\title{
Selective expansion of regulatory $T$ cells using an orthogonal IL-2/IL-2 receptor system facilitates transplantation tolerance
}

\author{
Toshihito Hirai, ${ }^{1,2}$ Teresa L. Ramos, ${ }^{1}$ Po-Yu Lin,, ${ }^{1}$ Federico Simonetta, ${ }^{1}$ Leon L. Su, ${ }^{3}$ Lora K. Picton, ${ }^{3}$ Jeanette Baker, ${ }^{1}$ \\ Jian-Xin Lin, ${ }^{4}$ Peng Li, ${ }^{4}$ Kinya Seo, ${ }^{5}$ Juliane K. Lohmeyer, ${ }^{1}$ Sara Bolivar-Wagers, ${ }^{6}$ Melissa Mavers, ${ }^{1,7}$ Warren J. Leonard, ${ }^{4}$ Bruce R. \\ Blazar, ${ }^{6}$ K. Christopher Garcia, ${ }^{3}$ and Robert S. Negrin ${ }^{1}$ \\ 'Division of Blood and Marrow Transplantation, Department of Medicine, Stanford University, Stanford, California, USA. Department of Urology, Tokyo Women's Medical University, Tokyo, Japan. \\ ${ }^{3}$ Departments of Molecular and Cellular Physiology and Structural Biology, Stanford University School of Medicine, Stanford, California, USA. ${ }^{4}$ Laboratory of Molecular Immunology and the Immunology \\ Center, National Heart, Lung, and Blood Institute, NIH, Bethesda, Maryland, USA. ${ }^{5}$ Division of Cardiovascular Medicine, Department of Medicine, Stanford University, Stanford, California, USA. ${ }^{6}$ Division of \\ Blood and Marrow Transplantation, Department of Pediatrics and the Masonic Cancer Center, University of Minnesota, Minneapolis, Minnesota, USA. ${ }^{7}$ Division of Pediatric Hematology, Oncology, Stem Cell \\ Transplantation and Regenerative Medicine, Lucile Packard Children's Hospital, Stanford University, Stanford, California, USA
}

\begin{abstract}
Adoptive transfer of Tregs has been shown to improve alloengraftment in animal models. However, it is technically challenging to expand Tregs ex vivo for the purpose of infusing large numbers of cells in the clinic. We demonstrate an innovative approach to engineering an orthogonal IL-2/IL-2 receptor (IL-2R) pair, the parts of which selectively interact with each other, transmitting native IL-2 signals, but do not interact with the natural IL-2 or IL-2R counterparts, thereby enabling selective stimulation of target cells in vivo. Here, we introduced this orthogonal IL-2R into Tregs. Upon adoptive transfer in a murine mixed hematopoietic chimerism model, orthogonal IL-2 injection significantly promoted orthogonal $\mathrm{IL}^{2}-\mathrm{R}^{+} \mathrm{Foxp}^{\mathrm{CFP}+} \mathrm{CD}^{+}$cell proliferation without increasing other $\mathrm{T}$ cell subsets and facilitated donor hematopoietic cell engraftment followed by acceptance of heart allografts. Our data indicate that selective target cell stimulation enabled by the engineered orthogonal cytokine receptor improves Treg potential for the induction of organ transplantation tolerance.
\end{abstract}

\section{Introduction}

Organ transplantation is a highly effective solution for end-stage organ failure. Current immunosuppressive agents successfully improve long-term graft survival. However, lifelong immunosuppression results in life-threatening comorbidities and poor efficacy in preventing chronic allograft rejection. Induction of mixed hematopoietic chimerism is one of the most reliable approaches to reproducibly achieving long-term allograft acceptance without maintenance immunosuppression in clinical kidney transplantation (1). However, it remains challenging to achieve donor hematopoietic stem cell (HSC) engraftment long term across MHC barriers with reduced intensity preconditioning.

In clinical trials of combined kidney and HSC transplant, patients who developed organ tolerance showed enrichment of Tregs at early time points after transplant (2-4). Therefore, it is hypothesized that Tregs play a pivotal role in mixed chimerism induction. Indeed, it has been demonstrated that Treg transfer promoted HSC engraftment in murine mixed chimerism models

Authorship note: TLR and PYL contributed equally to this work.

Conflict of interest: Ortho IL-2 is the subject of a pending patent (US patent number 10,869,887; KCG, LKP, and Jonathan T. Sockolosky). KCG is a founder of Synthekine. Copyright: (5) 2021, American Society for Clinical Investigation.

Submitted: May 7, 2020; Accepted: February 23, 2021; Published: April 15, 2021

Reference information: J Clin Invest. 2021;131(8):e139991.

https://doi.org/10.1172/JCl139991.
(5-11), implying that Treg transfer with the goal of mixed chimerism induction could be an effective strategy to achieve long-term organ transplantation tolerance. However, due to the paucity of Tregs and the likely requirement of large numbers of cells for establishing a tolerogenic milieu in nonmyeloablated hosts (12), exclusive transfer of Tregs alone is unlikely to be sufficient to achieve this goal.

IL-2 is essential for Treg generation, survival, stability, and function $(13,14)$. The IL-2 receptor complex (IL-2R) has 3 distinct subunits, designated IL-2R $\alpha$ (CD25), IL-2R $\beta$ (CD122), and common $\gamma$ chain $(\mathrm{CD} 132, \gamma \mathrm{c})$. Although CD25 does not participate in signaling, CD25/IL-2 binary complex increases the affinity of IL-2 for IL-2R $\beta$, then recruits $\gamma c$, leading to intracellular signaling in part via the JAK/STAT pathway. Since Tregs constitutively express CD25, they are more sensitive to IL-2 stimulation compared with the other immune cell populations, such as conventional $\mathrm{T}$ cells (Tcons) and NK cells, which express CD25 only when they are activated. Based on this discrepancy of IL-2 susceptibility, low-dose IL-2 therapy has been utilized for the treatment of patients with steroid refractory graft-versus-host diseases (GvHDs) or autoimmune diseases. Although substantial expansion of endogenous Tregs and therapeutic benefits have been observed (15-17), the impact of IL-2 on other cell populations can result in toxicities (18). IL-2/anti-IL-2 antibody complex or fusion protein has also been developed with the purpose of reducing affinity of IL-2 to IL-2R $\beta$ to selectively stimulate $\mathrm{CD}^{2} 5^{+}$Tregs $(19,20)$. In a murine BM 
transplantation (BMT) model, IL-2/anti-IL-2 complex administration ameliorated chronic GvHD upon combination with adoptive Treg transfer. However, in an acute GvHD model, IL-2/anti-IL-2 complex administration abrogated the therapeutic effect of adoptively transferred Tregs by expanding Tcons (21). IL-2/anti-IL-2 antibody complex also activated $\mathrm{NK}$ and $\mathrm{CD} 8^{+} \mathrm{T}$ cells in murine mixed chimerism induction models and caused engraftment failure (22). Those data collectively suggest that a more selective approach is required for enhancing Treg function for regulating immune reactions.

The orthogonal IL-2 (ortho IL-2) system was developed as a strategy for selectively expanding specific cell populations. This cytokine does not bind to WT IL-2R $\beta$ even in the presence of CD25, whereas it does bind to its specific engineered counterpart: ortho IL-2R $\beta$ (23-25). Prior work demonstrated the efficacy of this approach in Tcons with selective proliferation of ortho IL-2R $\beta$-transduced $\mathrm{T}$ cells in vivo following ortho IL-2 administration. Since the interaction of ortho IL-2 with ortho IL-2R $\beta$ is enhanced in the presence of CD25, we presumed that the ortho IL-2 system can more specifically and robustly contribute to Treg proliferation compared with that of Tcons. Here, we implemented ortho IL-2R $\beta^{+}$Treg adoptive transfer into a murine mixed hematopoietic chimerism induction model. We demonstrate that ortho IL-2 administration selectively expanded ortho IL-2R $\beta^{+}$Tregs, improved donor HSC engraftment, and led to organ transplantation tolerance.

\section{Results}

ortho IL-2R $\beta$-transduced Tregs show selective expansion by ortho $I L-2$ stimulation in vitro. Foxp $3^{\mathrm{GFP}+} \mathrm{BALB} / \mathrm{c}$ mice were utilized as Treg donors to purify Foxp $3^{+}$Tregs. Isolated Foxp $3^{\mathrm{GFP}+}$ Tregs were expanded as previously reported (26). The ortho IL-2R $\beta$ construct labeled with turboRFP (tRFP) was retrovirally transduced into Tregs during this expansion phase. After 1 week of culture, Tregs expanded $7.1 \pm 4.3$-fold (mean $\pm \mathrm{SD}$ ), with $30.8 \% \pm 12.3 \%$ transduction efficiency (Supplemental Figure 1, A-C; supplemental material available online with this article; https://doi.org/10.1172/ JCI139991DS1). Manipulated cells maintained Foxp3 $3^{\mathrm{GFP}}$ expression more than 99\% (Supplemental Figure 1D). The transduction process had no negative impact on cell expansion compared with cells cultured without viral vector (Supplemental Figure 1E). To validate the biological activity of cells transduced with tRFP/ortho IL-2R $\beta$, transduced Tregs were stimulated with WT or ortho IL-2 at the indicated concentrations. The expression level of CD25, inducible $\mathrm{T}$ cell costimulator (ICOS), and the proliferation of $\mathrm{tRFP}^{+}$and $\mathrm{tRFP}^{-}$fraction were compared (Figure 1A and Supplemental Figure 2). WT IL-2 stimulation resulted in upregulation of CD25 expression (Figure 1B) and promoted cell proliferation (Figure $1 \mathrm{C}$ ) in both $\mathrm{RFFP}^{+}$and $\mathrm{RFF}^{-}$fractions, indicating that transduced cells retain responsiveness to WT IL-2. Ortho IL-2 stimulation upregulated CD25 expression and promoted proliferation of the $\mathrm{RFP}^{+}$fraction with only minimal impact on the $\mathrm{RFP}^{-}$fraction (Figure 1, B-D). Ortho IL-2 stimulation also upregulated expression levels of ICOS, which endows Tregs with stronger suppressive function (27). To further assess the crossreactivity of ortho IL-2 on WT IL-2R, we stimulated WT Tregs that were expanded without viral vectors with ortho IL-2 (Supplemental Figure 3). WT Tregs did not respond to ortho IL-2 stimulation, demonstrating exquisite cytokine-receptor specificity. Ortho IL-2 was previously shown to be significantly less potent for STAT5 phosphorylation and cell growth compared with WT IL-2 for both Tcons and Tregs in vitro. In agreement, ortho IL-2 showed less potency to activate $\mathrm{RRFP}^{+}$ cells compared with WT IL-2 at low doses (Figure 1D: relative ratio to no IL-2 control at $1 \times 10^{3} \mathrm{IU} / \mathrm{mL}$ : CD25 expression level, $2.78 \pm$ 0.11 in WT IL-2, $2.23 \pm 0.11$ in ortho IL-2: $P=0.0039$; cell number: $4.32 \pm 0.41$ in WT IL-2, $2.25 \pm 0.05$ in ortho IL-2: $P=0.0115)$. However, ortho IL-2 increased the effect in a dose-dependent manner and showed comparable or greater effects than WT IL-2 at $1 \times 10^{5}$ $\mathrm{IU} / \mathrm{mL}$ (Figure 1D: relative ratio at $1 \times 10^{5} \mathrm{IU} / \mathrm{mL}$ : CD25 expression level, $2.76 \pm 0.34$ in WT IL-2, $4.05 \pm 0.02$ in ortho IL-2: $P=0.0220$; cell number: $8.96 \pm 3.16$ in WT IL-2, $8.10 \pm 0.36$ in ortho IL-2: $P=0.6836)$. Taken together, these studies demonstrated successful introduction of functional ortho IL-2R $\beta$ into Tregs without compromising expansion of these cells.

Ortho IL-2 stimulation does not abrogate Treg suppression in vitro. To confirm suppressive capacity of ortho IL-2R $\beta^{+}$Tregs after manipulation, Thy1.2+ Tregs that were transduced with ortho IL-2R $\beta$ were cocultured with naive Thy1.1 $1^{+}$Tcons that were activated with CD3/CD28 beads. Transduced Tregs were not purified and used as mixture of ortho IL-2R $\beta^{-}$and ortho IL-2R $\beta^{+}$ Tregs (defined as oTregs: $\sim 30 \%$ transduction efficiency). Four days later, the phenotypes of Thy1.1+ Tcons and oTregs were analyzed by flow cytometry (Supplemental Figure 4). oTreg coculture significantly repressed CD25 and ICOS expression on Thy1.1 $1^{+}$ Tcons and reduced the proliferation of Thy1.1 $1^{+}$Tcons, indicating that oTregs retain suppressive capacities (Figure 2, A-D). It has been reported that Tregs suppress $\mathrm{T}$ cell proliferation by IL-2 deprivation and that IL-2 replacement abrogates in vitro Treg suppression (28-30). In agreement with this, adding IL-2 restored CD25 and ICOS expression and proliferation of Thy1.1 ${ }^{+}$ Tcons (Figure 2, A-D). Conversely, ortho IL-2 did not affect CD25 expression on Thy1.1 $1^{+}$Tcons (Figure 2, A-D), whereas it resulted in an increase in CD25 expression levels on oTregs in a dosedependent manner (Figure 2E). Although ortho IL-2 at high doses $\left(1 \times 10^{5} \mathrm{IU} / \mathrm{mL}\right)$ slightly increased Thy1.1 $1^{+}$Tcon proliferation (Figure 2D, $P=0.077$ ), the suppressive function of oTregs was sustained $(P<0.001$ compared with Tcons alone). Meanwhile, the number of oTregs significantly increased by ortho IL-2 stimulation in a dose-dependent manner (Figure 2F). As a result, ortho IL-2 stimulation skewed the oTreg/Tcon ratio into a Tregdominant state (Figure 2G: \% oTregs: no IL-2, 42.6\% $\pm 6.0 \%$; ortho IL-2, $1 \times 10^{3} \mathrm{IU} / \mathrm{mL}, 55.7 \% \pm 3.4 \% ; 1 \times 10^{4} \mathrm{IU} / \mathrm{mL}, 61.3 \%$ $\pm 2.2 \% ; 1 \times 10^{5} \mathrm{IU} / \mathrm{mL}, 64.9 \% \pm 0.9 \% ; 1 \times 10^{6} \mathrm{IU} / \mathrm{mL}, 79.3 \% \pm$ $3.6 \%)$. Together, these data confirm that ortho IL-2 stimulation selectively stimulates oTregs in the presence of WT Tcons in vitro.

Ortho IL-2 stimulation of ortho IL-2R $\beta$ transduced Tregs transmits signals identical to those of native IL-2 signaling pathways. To understand the downstream pathway activation of ortho IL-2R $\beta^{+}$ Tregs following ortho IL-2 stimulation, we conducted transcriptome analysis after ortho IL-2 stimulation. First, we utilized a truncated human epidermal growth factor receptor-tagged (hEGFR-tagged) ortho IL-2R $\beta$ construct to enable the isolation of transduced cells with magnetic beads (Supplemental Figure 5, A and B). Consistent with the tRFP construct, enriched hEG- 
A

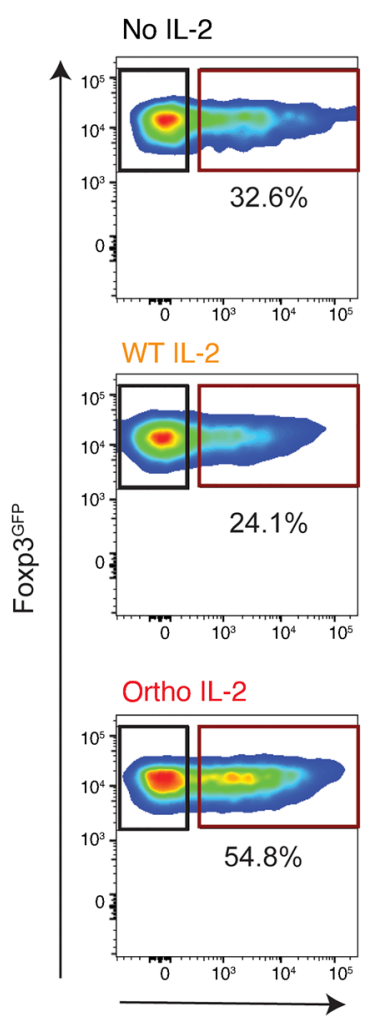

tRFP-ortho IL-2R $\beta$
B

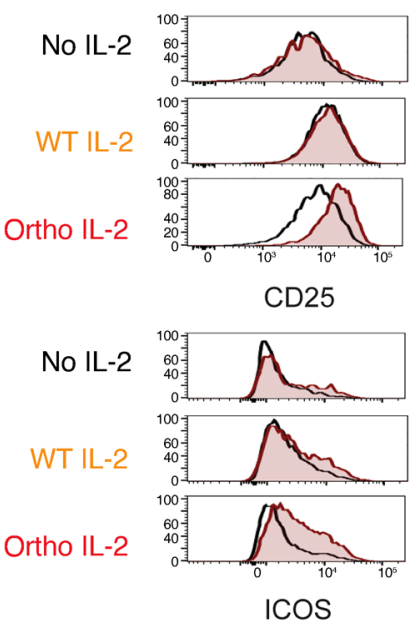

C

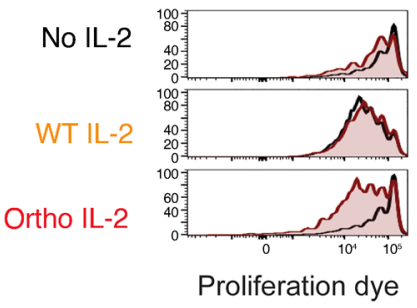

D
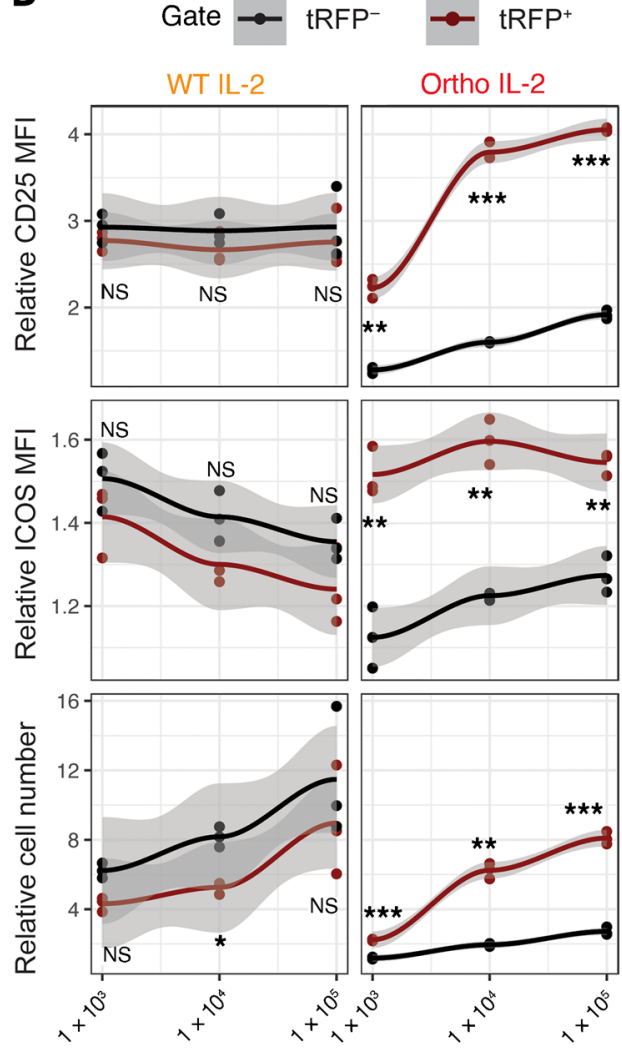

IL-2 concentration (IU/mL)

Figure 1. Ortho IL-2 stimulation selectively activates and expands Tregs with olL-2R $\beta$. Tregs transduced with tRFP/olL-2R $\beta$ were stained with violet cell proliferation dye, followed by incubation with $\mathrm{CD} 3 / \mathrm{CD} 28$ activation beads at a cell/bead 1:2 ratio. Flow cytometry analyses performed after 4 -day culture are shown. Representative pseudocolor plots (A) and histograms (B and C) of Tregs after incubation with no IL-2 (top), WT IL-2 (middle, $1 \times 10^{3} \mathrm{IU} / \mathrm{mL}$ ), or ortho IL-2 (bottom, $1 \times 10^{5} \mathrm{IU} / \mathrm{mL}$ ); brown gate: tRFP ${ }^{+}$fraction, black gate: tRFP- fraction. (D) Relative ratio to no IL-2 control in CD25 MFI, ICOS MFI, and cell number per well of each gated cell with mean plus $95 \%$ confidence intervals at each IL-2 concentration. Quantification of triplicate wells from 1 representative experiment of 3 independent experiments. ${ }^{*} P<0.05$; ${ }^{* *} P<0.01$; ${ }^{* *} P<0.001$, calculated between tRFP and tRFP-fraction by Welch's 2 -sample $t$ test. WT and ortho IL-2: $1 \mathrm{IU}=312.5 \mathrm{ng}$.

$\mathrm{FR}^{+}$Tregs reacted to ortho IL-2 stimulation in a dose-dependent manner (Supplemental Figure 5C). Enriched ortho IL-2R $\beta^{+}$Tregs were stimulated with WT or ortho IL-2 for 4 hours, and mRNA was extracted and analyzed by high-throughput RNA-Seq. Unsupervised principal component analysis (Figure 3A) demonstrated clustering of unstimulated control samples (no IL-2) away from WT or ortho IL-2-stimulated samples, while cluster of WT and ortho IL-2-stimulated samples overlapped. Pairwise comparison identified 1426 differentially expressed genes (DEGs) between no IL-2 and WT IL-2-stimulated samples and 1713 DEGs between no IL-2 and ortho IL-2 (Figure 3B), whereas no single genes passed the DEG thresholds $(\mathrm{FC}>1.5$ and $\mathrm{FDR}<0.05)$ when comparing WT and ortho IL-2 (Figure 3B). Indeed, 1340 DEGs overlapped between WT and ortho IL-2-stimulated cells (Figure 3C). See Supplemental Data Set 1 for full list of DEGs. Both WT and ortho IL-2-stimulated cells showed upregulation of IL-2dependent genes, such as Cish, Socs1, Socs2, and Lta (Figure 3, D and E, and ref. 31). Downregulation of Bcl6 was shown in both WT and ortho IL-2-stimulated cells in line with a published report that IL-2 signaling negatively regulates Bcl6 (32). WT and ortho IL-2-stimulated Tregs upregulated $B c l-2$ and $M y c$, which protect cells from apoptosis (33). Both populations of stimulated cells also expressed Tnfrsf18, Il2ra, Lilrb4, Tgfb1, Il10, and Foxp3, which are reported to be related to Treg immunosuppressive function (34). BioPlanet 2019 pathway analysis conducted by Enrichr $(35,36)$ demonstrated enrichment of DEGs in the pathways related to IL-2/STAT5 signaling in both WT and ortho IL-2-stimulated cells (Figure 3F), implying that ortho IL-2R $\beta$, together with $\gamma \mathrm{c}$, binds to JAK1 and JAK3 and leads to phosphorylation, which then recruits STAT5a/STAT5b $(14,37)$. These data collectively demonstrate that ortho IL-2/ortho IL-2R $\beta$ binding in Tregs elicits intracellular signaling through the STAT5 pathway.

Ortho IL-2 stimulation shows selective expansion of transferred Tregs in vivo. Anti-CD4OL $\mathrm{mAb}$ is considered a backbone of many nonmyeloablative mixed chimerism induction regimens in murine and primate models $(1,38)$. The advantage of anti-CD $40 \mathrm{~L}$ $\mathrm{mAb}$ is that blockade of the CD40/CD40L pathway obviates the need for global $\mathrm{T}$ cell deletion that is frequently used in other models to achieve alloengraftment (39), but that might also preclude expansion of adoptively transferred Tregs. It was also reported that anti-CD4OL mAb facilitated induction of Tregs involved in GvHD prevention and alloengraftment $(40,41)$. 
A

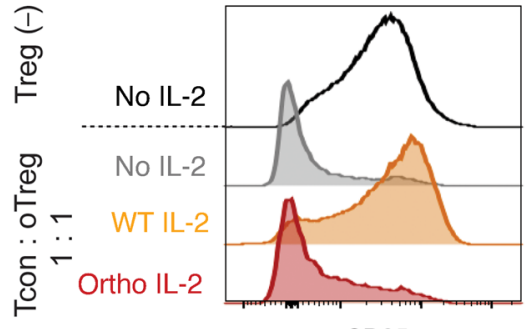

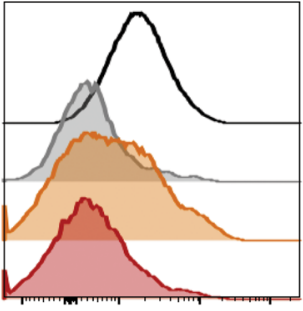

ICOS

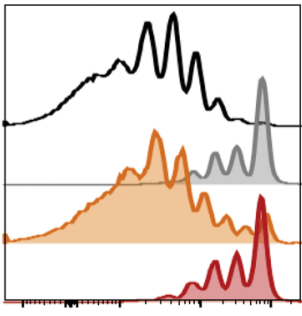

Proliferation dye

B

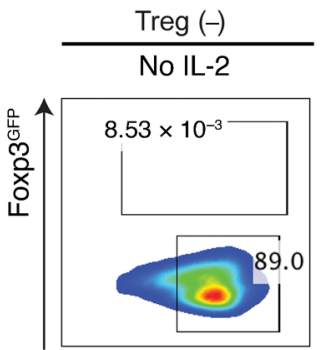

Tcon : oTreg 1:1

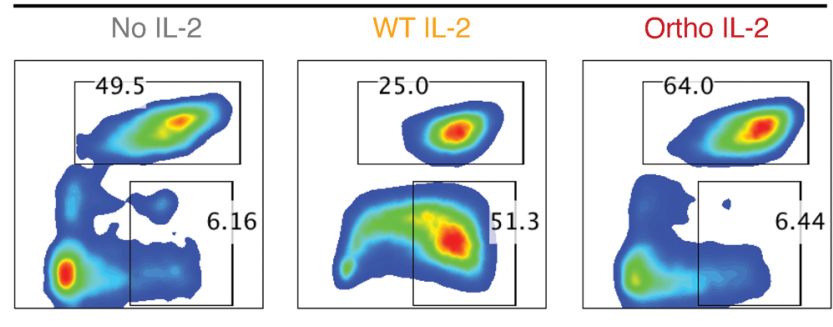

CD25

C

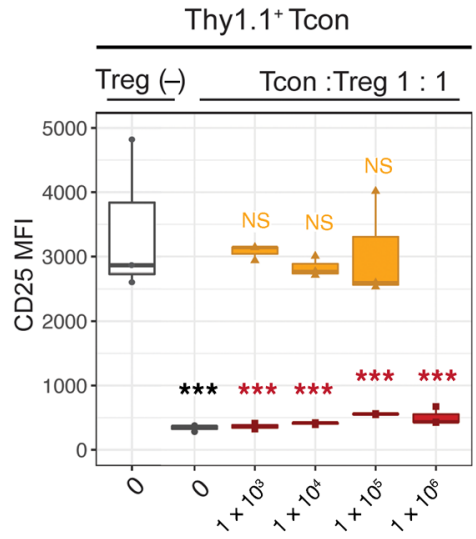

$\mathrm{IL}-2$ concentration (IU/mL)
D

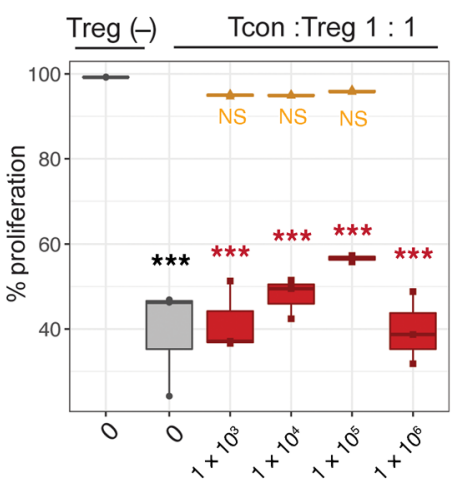

$\mathrm{IL}-2$ concentration (IU/mL)
E oTreg

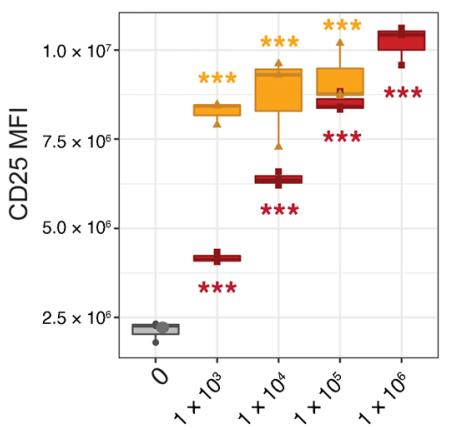

$\mathrm{IL}-2$ concentration (IU/mL)
$\mathbf{F}$

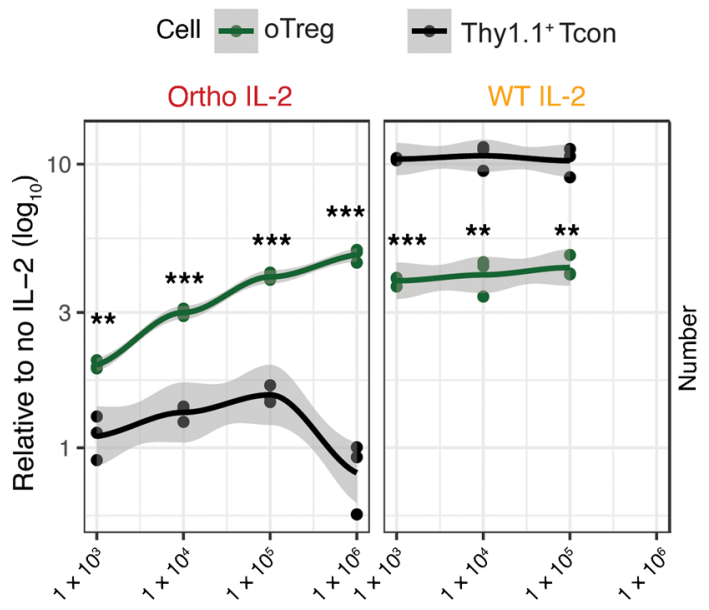

$\mathrm{IL}-2$ concentration $(\mathrm{IU} / \mathrm{mL})$
G

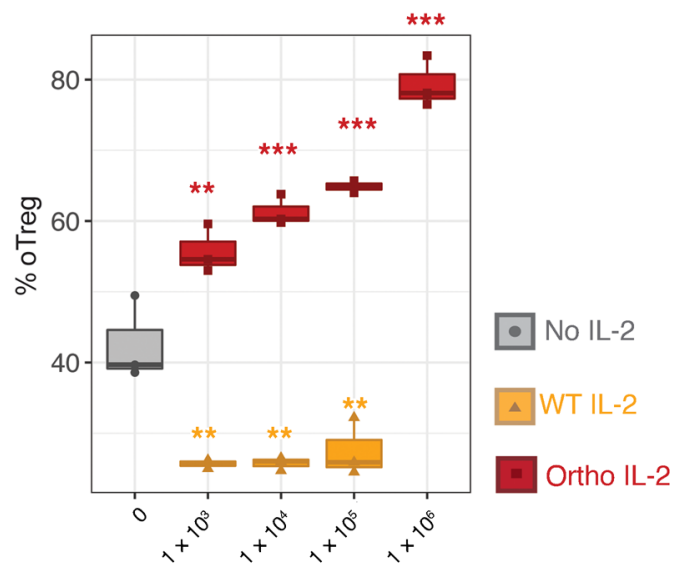

IL-2 concentration (IU/mL) 
Figure 2. Ortho IL-2 selectively expands olL-2R $\beta$-transduced Tregs without affecting cocultured naive $T$ cells or reducing Treg functions. Naive Thy1.1 ${ }^{+}$ Tcons were cocultured with Thy $1.2^{+}{ }^{+} \operatorname{Fxp}^{\mathrm{CFP}+}$ Tregs that were transduced with olL-2R $\beta$ (oTregs; transduction efficiency was $~ 30 \%$ ). Flow cytometry analyses performed after 4-day coculture. (A) Representative histograms show expression levels of CD25 and ICOS and dilution of proliferation dye on Thy $1.1^{+}$Tcons. (B) Representative pseudocolor plots show the propor-

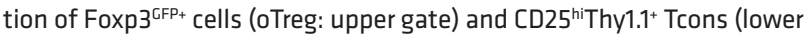
gate). Gray, no IL-2; orange, WT IL-2 $\left(1 \times 10^{3} \mathrm{IU} / \mathrm{mL}\right)$; red, ortho IL-2 $\left(1 \times 10^{5}\right.$ $\mathrm{IU} / \mathrm{mL}$ ). Box plots showing CD25 MFI (C) and percentage of proliferation (D) in Thy $1.1^{+}$Tcons. Thy $1.1^{+}$Tcon alone is shown as no suppression control (Treg [-], white box). $P$ values in yellow/red on the columns were calculated between Tcon alone control and coculture at each IL-2 concentration. (E) Box plots showing CD25 MFI in oTregs. (F) Cell number per well relative to no IL-2 control in Thy $1.1^{+}$Tcons (black line) or oTregs (green line) with mean plus $95 \%$ confidence intervals. $P$ values calculated between Thy $1.1^{+}$Tcons and oTreg at each IL-2 concentration are shown. (G) Box plots showing percentage of oTregs per well. $P$ values were calculated between no IL-2 control and each IL-2 concentration. Quantification of triplicate wells in 1 representative experiment of 3 independent experiments. ${ }^{*} P<0.01$; ${ }^{* *} P<0.001$ Welch's 2-sample $t$ test. WT and ortho IL-2: $1 \mathrm{IU}=312.5 \mathrm{ng}$.

To evaluate in vivo effect of ortho IL-2 in this system, we transferred oTregs into Thy $1.1^{+} \mathrm{BALB} / \mathrm{c}$ mice that underwent $3.3 \mathrm{~Gy}$ total body irradiation followed by i.p. injection of $0.3 \mathrm{mg}$ anti-CD40L mAbs (Figure 4A). Thereafter, recipient mice were injected with PBS, WT IL-2, or ortho IL-2 once a day, up to day 6 (d6). The Treg phenotype in the spleen on d6 is shown in Figure $4, \mathrm{~B}$ and C. Consistent with the in vitro experimental data (Figure 2), ortho IL-2 selectively activated the $\mathrm{RFP}^{+}$fraction, but not the tRFP $^{-}$fraction in oTregs (tRFP ${ }^{+}$vs. $\mathrm{tRFP}^{-}$for CD25, $P=0.0006$; for ICOS, $P=0.0006$; Figure 4, B-D). Although WT IL-2 stimulation increased the number of oTregs in both $\mathrm{RFP}^{+}$and $\mathrm{tRFP}^{-}$fractions, it also increased Thy1.1 ${ }^{+}$host cells $3.98 \pm 1.13$-fold higher over the PBS group (Figure 4E); this was accompanied by upregulation of CD25 that might be exploited for allograft rejection (Figure 4, F and G, and Supplemental Figure 7). In contrast, ortho IL-2-treated mice showed significant increases in the number of $\mathrm{tRFP}^{+}$oTregs $(3.68 \pm 1.81$-fold higher over those treated with PBS, $P=0.0058$ ), whereas the number of $\mathrm{tRFP}^{-}$oTregs and that of Thy $1.1^{+}$host cells were not altered (Figure 4E). Consequently, the proportion of total oTregs among host CD $45.2^{+}$lymphocytes became significantly higher in ortho IL-2-treated animals (ortho IL-2 vs. PBS, $P=0.0267$; ortho IL-2 vs. WT IL-2, $P=0.0397$ ) (Figure $4, \mathrm{~F}$ and $\mathrm{G})$. Despite significant expansion, all Thy $1.2^{+}$transferred populations sustained Foxp $3^{\mathrm{GFP}}$ expression (Supplemental Figure 6), implying stability of the Treg phenotype after the treatment. Ortho IL-2-treated mice also showed a significant increase in Foxp ${ }^{\mathrm{GFP}+}$ Tregs in total body blood $(24.6 \% \pm 16.7 \%$ in PBS, $58.7 \% \pm 7.0 \%$ in ortho IL-2; $P=0.02857)$ and peripheral lymph nodes $(4.30 \% \pm 1.33 \%$ in PBS, $7.10 \% \pm 1.93 \%$ in ortho IL- $2 ; P=$ 0.01107 ; Figure $4 \mathrm{H})$. These data demonstrate that ortho IL-2 can selectively expand transduced Tregs in vivo.

oTreg transfer and ortho IL-2 treatment synergistically enhances donor hematopoietic cell engraftment. To evaluate the effect of ortho IL-2 on BM engraftment, we continued daily WT and ortho IL-2 administration until d14 after oTreg transfer utilizing the same model shown in Figure 4A. Ortho IL-2-treated mice sustained the Foxp $3^{\mathrm{GFP}+}$ population at significantly higher levels $(9.29 \% \pm$
4.66\%) compared with the other treatment groups at d14 (PBS, $4.24 \% \pm 1.62 \%, P=0.0051$; WT IL-2, $4.00 \% \pm 1.70 \%, P=0.0046$; Figure 5, A and B), suggesting ortho IL-2 prolongs the survival of transferred Tregs. WT IL-2 significantly increased the proportion of $\mathrm{CD}^{+} \mathrm{T}$ cells $(36.9 \% \pm 15.4 \%)$, whereas ortho IL-2 did not alter this population compared with the PBS-treated group (PBS, $4.54 \% \pm 1.66 \%$; ortho IL-2, $3.43 \% \pm 1.64 \% ; P=0.286$ ), indicating the absence of detectable off-target effects in ortho IL-2-treated mice. To validate receptor-cytokine selectivity, we transferred expanded Foxp $3^{\mathrm{GFP}+}$ Tregs without transduction (untransduced [UT] Tregs) and treated them with ortho IL-2. As expected, ortho IL-2 did not increase Foxp $3^{\text {GFP+ }}$ UT Treg populations (Figure 5, A and $\mathrm{B})$. Donor cell chimerism was assessed as the percentage of donor $\mathrm{H} 2 \mathrm{~K}^{\mathrm{b}+} \mathrm{CD} 45.1^{+}$cells in PBMCs on d14 (Figure $5 \mathrm{C}$ ). Without Treg transfer, no mice showed donor cell chimerism (defined as donor cells $>1 \%$ ) regardless of IL-2 administration. The impact of Treg transfer alone on donor cell chimerism was not statistically significant compared with the no Treg transferred control group in both UT and oTregs (\% donor cells on d14: UT Treg + PBS, 6.57\% $\pm 20.8 \%, P>0.9999$; oTreg + PBS, $6.18 \% \pm 16.2 \%, P=$ $0.6004)$. Ortho IL-2 injection on UT Tregs did not improve donor cell chimerism $(5.12 \% \pm 15.3 \%, P=0.1999)$. Importantly, mice that received oTregs plus ortho IL-2 administration showed a significantly larger percentage of donor cells compared with the no Treg transferred control group $(11.2 \% \pm 16.7 \%, P=0.0002$; Figure $5 \mathrm{C})$. Although the proportion of Foxp $3^{\mathrm{GFP}+}$ Tregs returned to baseline levels 14 days after ortho IL-2 cessation (on d28, Supplemental Figure 8A), donor BMC engraftment was sustained in the mice that received oTregs + ortho IL-2 administration on d28 (Supplemental Figure $8 B ; 11.8 \% \pm 15.7 \%, P=0.0002$ compared with no Treg control animals) and donor cell chimerism was greater than $1 \%$ thereafter (Supplemental Figure $8 \mathrm{C}$ ). Success rate of chimerism induction on $\mathrm{d} 28$ ( $>1 \%$ donor cell chimerism) was $60 \%$ in oTregs plus ortho IL-2 administration group compared with $10 \%$ in those plus PBS administration group.

We also tested mixed chimerism induction in C57BL/6 mice as recipients and $\mathrm{BALB} / \mathrm{c}$ mice as donors to evaluate the ortho IL-2 effect on NK cells (Supplemental Figure 9A). The facilitation of engraftment and Treg-specific expansion was observed in the ortho IL-2-treated group (Supplemental Figure 9, B and C). WT IL-2 increased the proportion of CD62L-KLRG1 ${ }^{+} \mathrm{NK} 1.1^{+}$cells that are the effector NK cell phenotype (42), whereas ortho IL-2 did not alter NK cell populations (Supplemental Figure 9, D and E, and Supplemental Figure 10). Chimerism induction on d28 in C57BL/6 mice was $44 \%$ (4 out of 9) in the oTreg plus ortho IL-2 group, but only $11 \%$ (1 out of 9 ) in the PBS group.

Ortho IL-2 injection does not interfere with early donor cell distribution and prevents rejection. To evaluate the effect of IL-2 on the early phase of donor cell distribution, we utilized $l u c^{+} \mathrm{C} 57 \mathrm{BL} / 6$ mice as $\mathrm{BM}$ donors and quantified donor cell distribution by bioluminescent imaging (BLI), as previously described (43). Because total flux of photons in BLI reflects the number of engrafted $l u c^{+}$cells, it represents donor cell distribution more sensitively and accurately compared with flow cytometry, especially at the early time points when the numbers of donor-derived PBMCs are low. Indeed, photons on $\mathrm{d} 4$ were localized to bones and the spleen, but not distributed in 
A

Treatment No IL-2 WT IL-2 Ortho IL-2

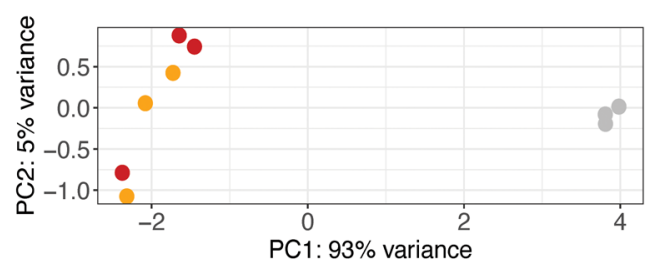

B

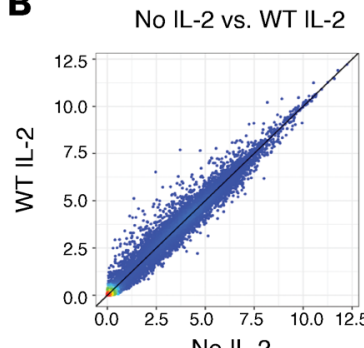

No IL-2

C

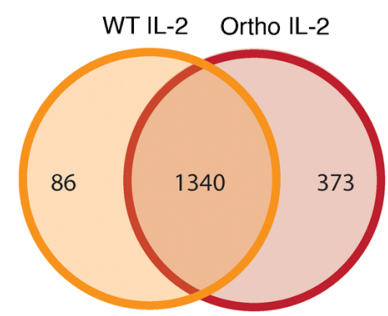

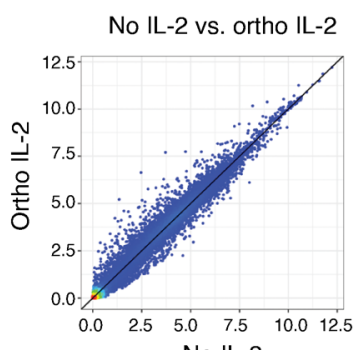

No IL-2

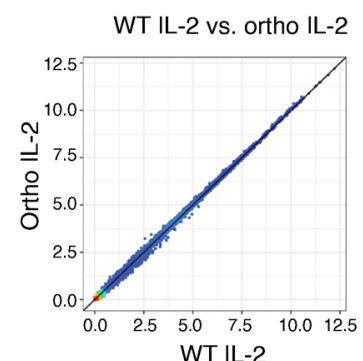

WT IL-2
E

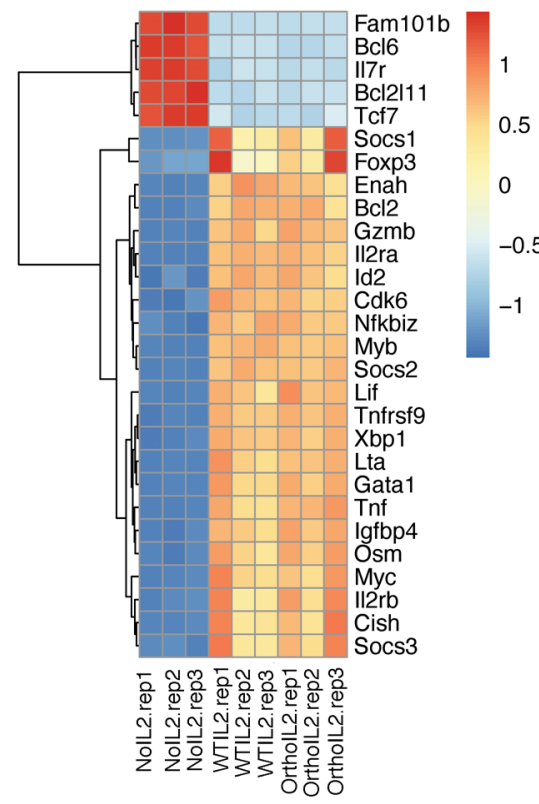

D

WT IL-2

Ortho IL-2
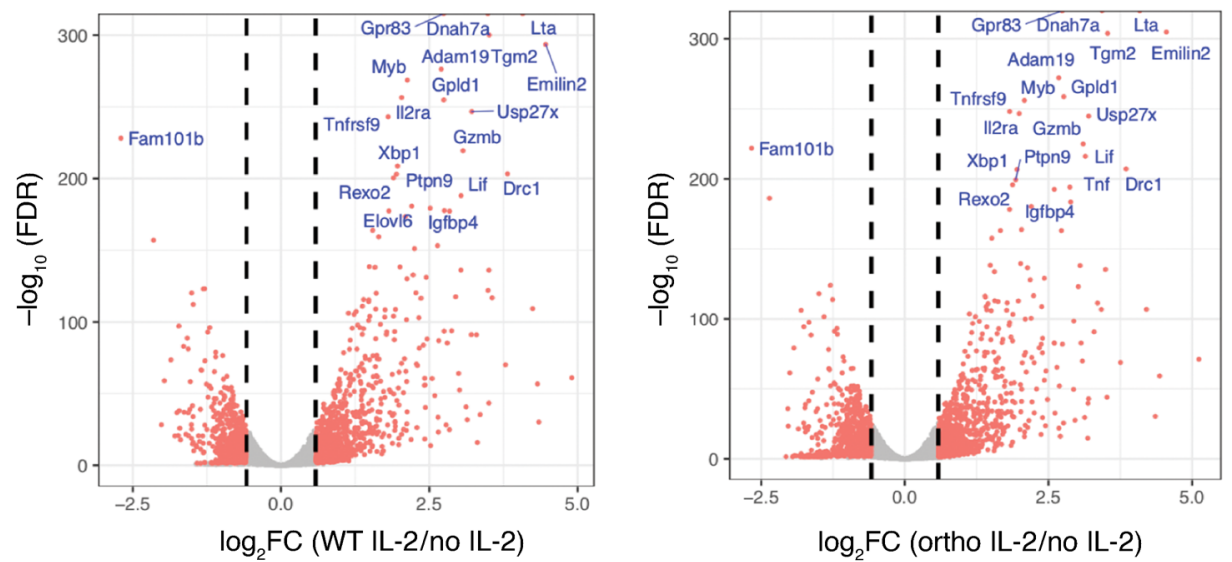

$\mathbf{F}$

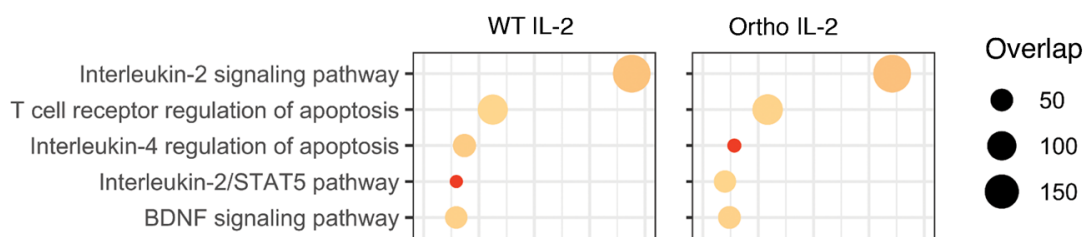
Immune system Insulin signaling pathway Interleukin-2 receptor beta chain in T cell activation Immune system signaling by interferons, interleukins, prolactin, and growth hormones Cytosolic tRNA aminoacylation Jak-STAT signaling pathway Prolactin regulation of apoptosis TGF-beta regulation of extracellular matrix
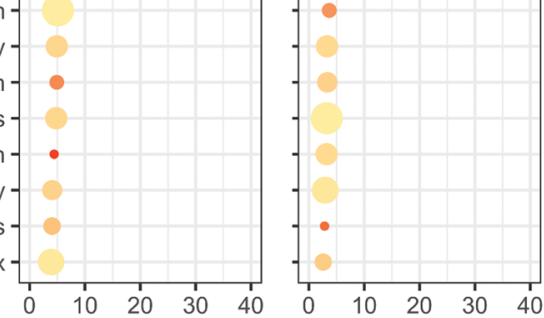

Enrich_factor

$=0.5$

0.4

0.3

0.2

$-\log _{10}$ (adjusted $P$ value) 
Figure 3. RNA-Seq reveals upregulation of transcripts involved in IL-2 signaling after ortho IL-2 stimulation. $\mathrm{Foxp}^{\mathrm{CFP}_{+}}$Tregs were transduced with hEGFR/olL-2R $\beta$. hEGFR ${ }^{+}$cells were enriched by magnetic cell isolation using an anti-hEGFR mAb. Enriched cells were restimulated with WT or ortho IL-2 for 4 hours after overnight IL-2 starvation. (A) Principal component analysis revealed clustering of WT- (orange) and ortho IL-2-stimulated (red) samples away from unstimulated control cells (no IL-2, gray). (B) Scatterplot of mean RPKM values $\left(\log _{2}\right)$ in no IL-2 vs. WT IL-2 (left), no IL-2 vs. ortho IL-2 (middle), and WT IL-2 vs. ortho IL-2 (right). (C) Venn diagram showing overlap of DEGs in WT and ortho IL-2-stimulated samples. (D) Volcano plots reveal DEGs in WT- (left) or ortho IL-2-stimulated (right) cells compared with no IL-2. Vertical dashed lines on volcano plots indicate a fold change of \pm 1.5 . The top 20 most differentially expressed genes are indicated. (E) Heatmap displaying DEGs in IL-2 signaling signature genes among no IL-2-, WT IL-2-, and ortho IL-2-stimulated samples. (F) The top 13 pathways with the smallest adjusted $P$ values in WT IL-2-stimulated cells shown in the BioPlanet 2019 pathway analysis. Pathway analysis of DEGs shown in ortho IL-2-treated cells reveals the same pattern as shown with WT IL-2-treated cells. Plots show the adjusted $P$ values, circle area represents the number of DEGs overlapping with genes in a given pathway, and circle color represents the rich factor reflecting the proportion of DEGs in a given pathway. Data shown are from 1 representative experiment from 3 independent experiments with biological triplicates.

the periphery, indicating that early distribution of donor cells may be difficult to detect by evaluating PBMCs (Figure 5D). WT IL-2 administration significantly reduced total photon flux on $\mathrm{d} 4$ compared with PBS (Figure 5E, $P=0.0041$ ), indicating that WT IL-2 accelerated BM rejection. On the other hand, ortho IL-2 administration did not interfere with donor cell distribution ( $P=0.5254$ vs. PBS). oTreg transfer plus PBS recipients showed higher total photon flux compared with no Treg transfer plus PBS recipients on $\mathrm{d} 10(P=0.0007)$ and $\mathrm{d} 28(P=$ 0.0021 ; Figure $5 \mathrm{~F}$ ), indicating engraftment-facilitating effect of oTregs. Moreover, compared with oTreg plus PBS recipients, oTreg plus ortho IL-2 recipients tended to show greater increases in total photon flux on $\mathrm{d} 10(P=0.0892)$ and revealed a significant increase on $\mathrm{d} 28(P=0.0288)$, suggesting that ortho IL-2 enhanced the potency of oTregs to prevent late-phase BM rejection that is thought to be driven by alloantigen-primed $\mathrm{T}$ cells. Supplemental Figure 11 shows time course of total photon flux for individual mice with multiple comparisons of each treatment group compared with no Treg control group at each time point. Consistent with flow cytometry results, UT Treg transfer with or without ortho IL-2 and oTreg transfer plus PBS did not show statistically significant improvement of the engraftment over the control group, whereas oTreg transfer plus ortho IL-2 significantly improved engraftment on $\mathrm{d} 10$ and d28. The mice showed sustained mixed chimerism (total flux > $10^{7}$ ) over 100 days after BMT (data not shown).

Mice with mixed chimerism accepted allogenic heart transplants from BM donors. To confirm the establishment of tolerance, we transplanted heart grafts obtained from luciferase ${ }^{+} \mathrm{H} 2^{\text {b+ }}$ donor into the ear pinna of recipient mice 2 months after BMT. To address antigen specificity, we transplanted third-party $\mathrm{H} 2^{9+}$ heart grafts on the opposite ear pinna of mice consecutively after $\mathrm{H}^{{ }^{\mathrm{b}}}$ graft acceptance (Figure 6, A-C). Heart-graft survival was monitored by BLI over time (Figure 6A). Tissue viability was verified by electrocardiogram (Supplemental Fig- ure 12). Nonchimeric mice that rejected $\mathrm{H} 2 \mathrm{~b}^{+}$donor BMCs by d56 eventually rejected $\mathrm{H} 2 \mathrm{~b}^{+}$heart allografts, whereas chimeric mice that had sustained $\mathrm{H} 2 \mathrm{~b}^{+}$donor cell engraftment until d56 at the time of heart transplantation accepted donor-derived heart allografts for 90 days (Figure 6B and Supplemental Figure 13). PBS plus oTreg transfer recipients tended to show greater survival compared with PBS without Treg control animals $(P=$ 0.0596). Animals that received ortho IL-2 plus oTreg adoptive transfer showed significant improvement in donor-derived heart allograft survival $(P=0.0003$ compared with PBS without Treg control), indicating that ortho IL-2 is capable of improving the function of oTregs for tolerance induction.

The third-party $\mathrm{H}^{\mathrm{q}+}$ graft was rejected in the same manner between chimeric and nonchimeric mice, demonstrating that chimeric recipients are immunocompetent for nondonor antigens. In vitro mixed lymphocyte reaction (MLR) also showed competent $\mathrm{T}$ cell reactions against $\mathrm{H} 2^{9^{+}}$cells in all treatment groups (Figure 6, D and E, and Supplemental Figure 14). Conversely, T cell reactivity against $\mathrm{H}^{\mathrm{b}+}$ cells was significantly reduced in the mice that underwent BMT with oTreg transfer plus ortho IL-2 administration compared with those with oTreg transfer plus PBS administration $(P=0.0279)$.

To elaborate on the relationship between early donor BM engraftment and achievement of organ tolerance, we plotted a receiver operating characteristic (ROC) curve for predicting long term donor-derived heart graft survival ( $>90$ days) by total flux in BMC-BLI at the early time points (Supplemental Figure $15 \mathrm{~A})$. We found that donor cell engraftment at $\mathrm{d} 7$ predicted heart transplantation tolerance (AUC, 0.940; cut-off total flux, $1.1 \times$ $10^{7}$ ). Since total photon flux by BLI on $\mathrm{d} 7$ and the percentage of Foxp $3^{\mathrm{GFP}} / \mathrm{CD} 4$ cells on $\mathrm{d} 14$ showed positive correlations (Supplemental Figure 15B, $R=0.57$ ), it was postulated that ortho IL-2 facilitated achievement of tolerance through the expansion of transferred Tregs. The positive correlation between early Treg expansion and donor cell chimerism development was also shown in the C57BL/6 model (Supplemental Figure 9, F and G). Taking these data together, we concluded that oTreg plus ortho IL-2 combination therapy has the potential to facilitate the development of donor-specific transplantation tolerance.

\section{Discussion}

The utilization of the ortho IL-2/IL-2R pair results in the exquisite selectivity of activating and expanding cell populations harboring the receptor. We previously reported that ortho IL-2 administration delayed tumor growth when it was combined with tumorspecific Tcons transduced with ortho IL-2R $\beta$ (23). Because ortho IL-2 is capable of stimulating target cells without other cells competing for this resource, the efficacy of tumor-growth suppression and survival benefits of ortho IL-2 was superior to that of WT IL-2 even when WT IL-2 was administered at a dose 10 times higher. We have now investigated a paradigm of the ortho IL-2/ ortho IL-2R system with the goal of enhancing immune regulation: a facet of the immune system that needs more delicate control. In this report, we assessed the impact of ortho IL-2 on the immune-suppressive potential of ortho IL-2R $\beta^{+}$Tregs. Whereas WT IL-2 increased non-Treg subsets and resulted in rapid BM rejection, ortho IL-2 showed no impact on off-target populations, 
A

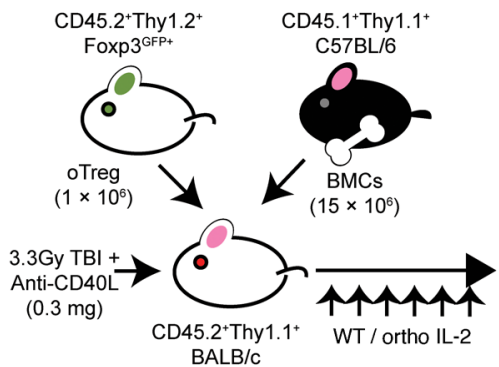

E

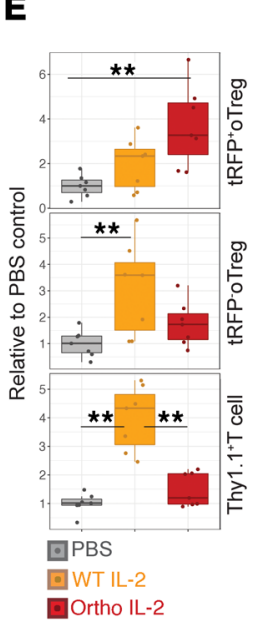

B

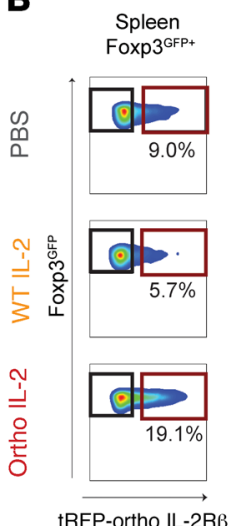

$\mathbf{F}$

tRFP-ortho IL-2RP
C
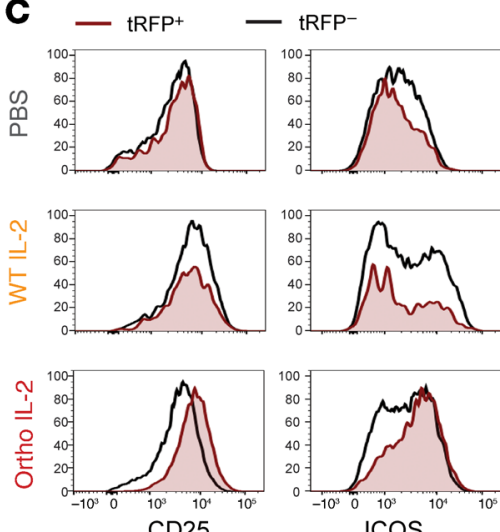

CD25
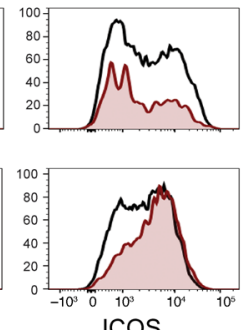

D $\quad$ TRFP-

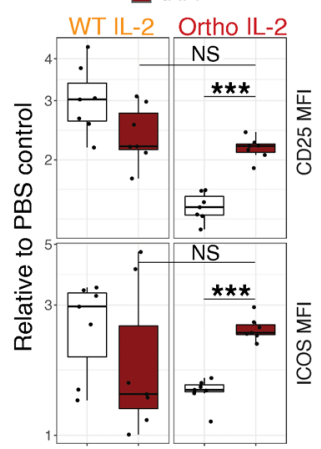

G

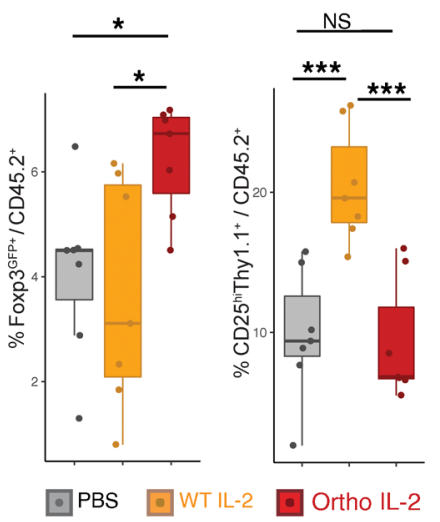

H

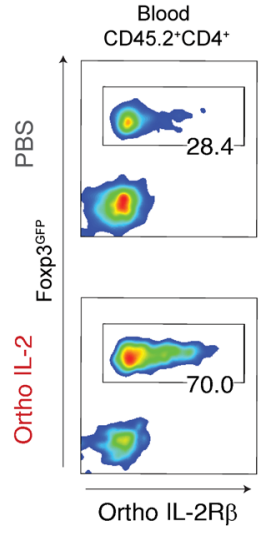

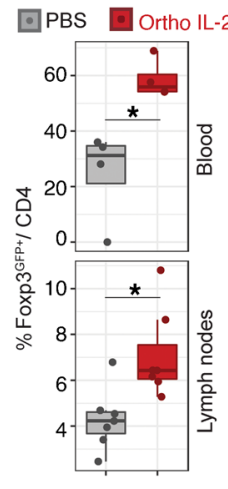

Figure 4. In vivo injection of ortho IL-2 selectively expands olL-2R $\beta$-transduced Tregs. (A) Schematic of in vivo model for mixed chimerism induction.

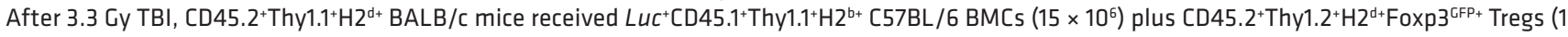
$\left.\times 10^{6}\right)$ that were transduced with olL-2R $\beta$ (oTreg: transduction efficiency was about 30\%). All mice were treated with anti-CD40L (0.3 mg i.p. on d0) followed by i.p. administration of PBS ( $n=7)$, WT IL-2 MSA 25,000 IU/d ( $n=7)$, or ortho IL-2 MSA 25,000 IU/d ( $n=7)$ for 6 days. Spleen, blood, and peripheral lymph nodes were recovered and analyzed by flow cytometry on d6. (B-D) Ortho IL-2 administration increases the population (B) and expression levels of CD25 and ICOS (C and D) on tRFP-positive fraction (brown gate), but not on tRFP-negative fraction (black) of oTregs in the spleen. (E) Box

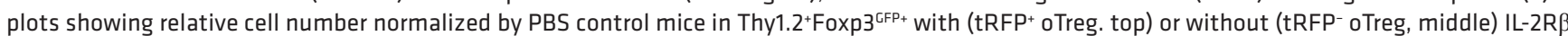

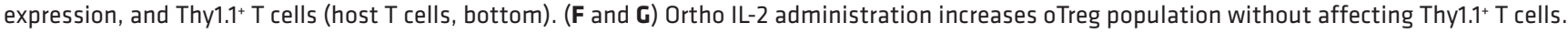
Representative pseudocolor plots of $\mathrm{CD} 45.2^{+}$splenocytes (F) and box plots (C). (H) The percentage of oTregs in CD45.2+CD4+ $4^{+}$cells in total body blood collected from the inferior vena cava and peripheral lymph nodes. Left, representative pseudocolor plots in blood. Right, comparison between PBS- and ortho IL-2-treated mice. Pooled data from 2 independent experiments including 3 to 4 mice per group per each experiment. ${ }^{*} P<0.05$; ${ }^{* *} P<0.01 ;{ }^{* *} P$ $<0.001$, between indicated groups calculated by Mann-Whitney $U$ test for 2-group comparison, Dunn's Kruskal-Wallis test for multiple group comparison. TBI, total body irradiation; CD4OL, CD40 ligand.

but increased ortho IL-2R $\beta^{+}$Tregs. Ortho IL-2 treatment facilitated BM engraftment and substantially enhanced donor-specific organ transplantation tolerance, demonstrating that the ortho IL-2 approach is capable of selectively enhancing Treg biological effects with potential clinical benefits.

RNA-Seq analysis demonstrated that ortho IL-2 stimulation upregulated genes involved in STAT5 signaling, including antiapoptotic genes $(\mathrm{Bcl}-2$ and $\mathrm{Myc}$ ) and Treg-activation genes (Tnfrsf18, Il2ra, Lilrb4, Foxp3), indicating that ortho IL-2 stimulation likely enhances ortho IL-2R $\beta^{+}$Treg survival and activation. Ortho IL-2 stimulation also upregulated Gzmb, which is indispensable for contact-mediated suppression and skin-transplantation tolerance induced by Tregs $(44,45)$. It is reported that IL-2-mediated STAT5 signaling also contributes to Treg-suppressive functions $(46,47)$. In line with that, ortho
IL-2-stimulated cells showed upregulation of genes critical for Treg immune suppression, including soluble factors, such as TGF- $\beta$ (Tgfb1) and IL-10 (Il10), and cell surface molecules, such as OX40 (Tnfrsf4), GITR (Tnfrsf18), and ILT3 (Lilrb4). Upregulation of other important surface molecules, such as CTLA-4 (Ctla4) or ICOS (Icos), was not observed, which may be due to the fact that transcripts were evaluated only 4 hours after stimulation to analyze the direct influence of ortho IL-2 on the downstream cascade of STAT5. Indeed, after 4-day incubation with ortho IL-2, ortho IL-2R $\beta^{+}$Tregs showed upregulation of ICOS, which is the surrogate marker for production of IL-10 and TGF- $\beta$ $(48,49)$. Similarly, genes for cell cycling or DNA replication were not observed in DEGs analyzed at 4 hours, though we observed promotion of cell proliferation in ortho IL-2-stimulated cells after 4 day culture. Taking these data together, we presume that 
A
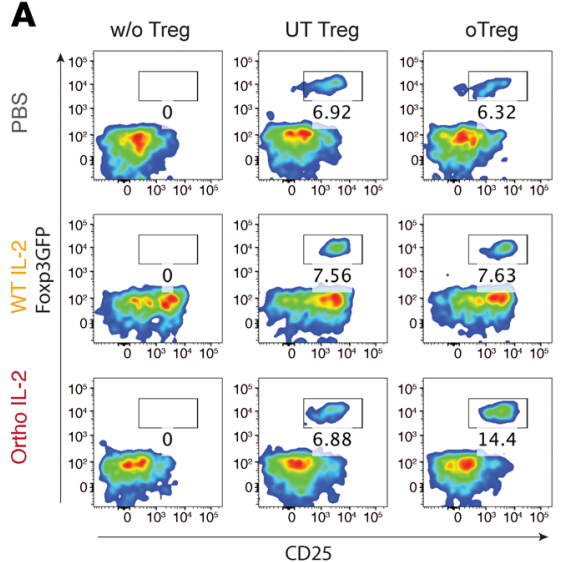

B
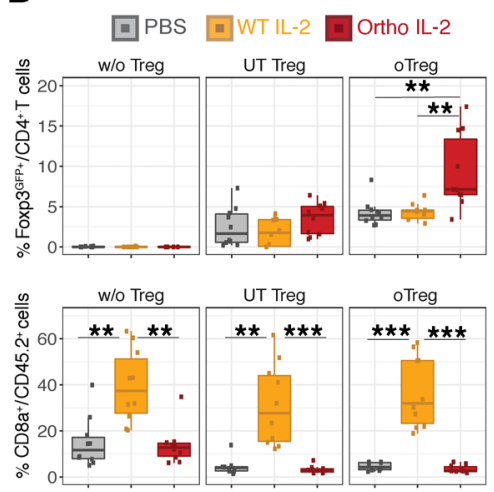

C

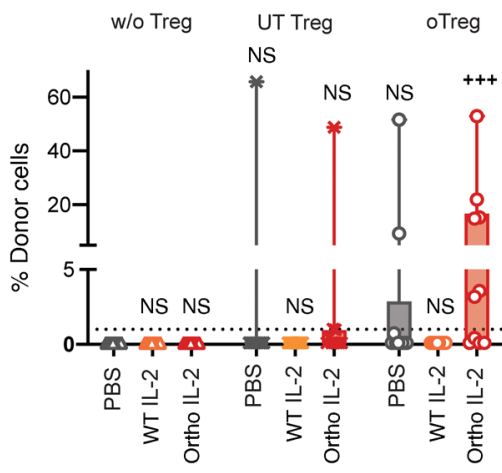

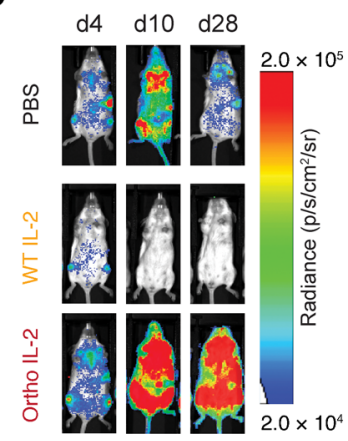

E

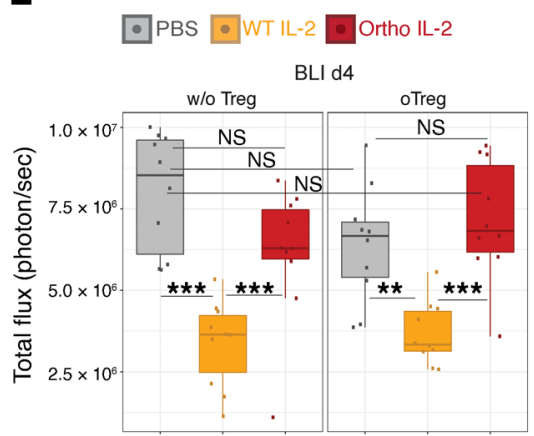

F

- PBS Dortho IL-2 BLI d10

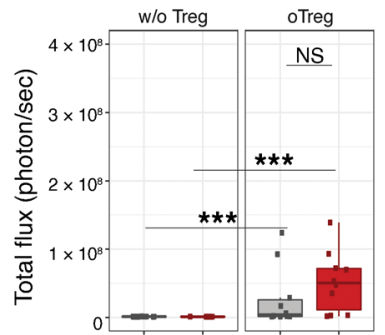

- PBS Ortho IL-2 BLI d28

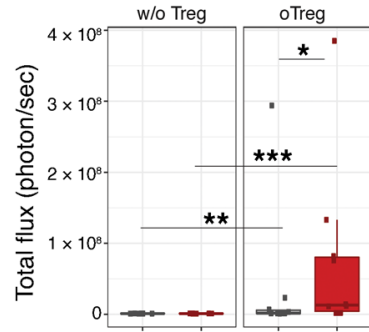

Figure 5. Ortho IL-2 stimulation increases transferred oTreg population and improves donor cell engraftment. After $3.3 \mathrm{~Gy} T B I, C D 45.2^{+}+2^{d+}$ WT $B A L B / C$ mice received $L u C^{+} C D 45.1^{+} \mathrm{H}^{\mathrm{b}+} \mathrm{C}$ C57BL/6 BMCs $\left(15 \times 10^{6}\right)$ plus CD45.2+ $2^{\mathrm{d}+} \mathrm{Foxp}^{\mathrm{CFP}+}$ Tregs $\left(1 \times 10^{6}\right)$ that were transduced with olL-2R $\beta$ (oTreg: transduction efficiency was $\sim 30 \%$ ) or those expanded without viral vector (UT Tregs). All mice were treated with anti-CD4OL (0.3 mg i.p. on d0), followed by i.p. administration of PBS, WT IL-2 MSA, or ortho IL-2 MSA (both IL-2, 25,000 IU/d) for 14 days. Flow cytometry analysis for PBMCs obtained from the tail vein was performed on d14. (A) Representative pseudocolor plots gated by CD45.2+ $2_{2} \mathrm{k}^{\mathrm{d}+C D 4^{+} \mathrm{T}}$ cells. (B) Box plots showing the percentages of Foxp $3^{\mathrm{CFP}+} \mathrm{CD} 4^{+}$(top) and CD8 ${ }^{+} \mathrm{CD} 45.2^{+}$cells (bottom). (C) Box and whiskers with minimum to maximum showing percentages of H2K ${ }^{\mathrm{b}+} \mathrm{CD} 45.1^{+}$donor cells in PBMCs on d14. Horizontal dotted line indicates $1 \%$ of donor cells. ${ }^{++} P<0.001$, comparing the mean rank of each column to the mean rank of PBS control without Tregs (gray triangle) by Dunn's Kruskal-Wallis multiple comparisons. (D) Representative bioluminescent images for mice that received oTregs showing distribution of engrafted BMCs. Box plots showing total photon flux on d4 (E), d10, and d28 (F). Pooled data from 2 independent experiments including 5 mice per group per each experiment. ${ }^{*} P<0.05$; ${ }^{* *} P<0.01$; ${ }^{* *} P<0.001$, between indicated 2 groups calculated by Dunn's Kruskal-Wallis multiple comparisons among the 3 treatment groups or by Mann-Whitney $U$ test between 2 groups.

ortho IL-2 potently provides a variety of signals for cell proliferation, survival, and immune regulation of Tregs.

Tregs bind IL-2 with high affinity due to constitutive expression of CD25. On the basis of this, many preclinical and clinical trials have tested IL-2-based therapies with the goal of preferential Treg activation. In the mixed chimerism induction models, Shatry et al. demonstrated that IL-2/anti-IL-2 antibody complex injection after transplant increased Tregs, inhibited early expansion of anti-donor-specific CD8 ${ }^{+} \mathrm{T}$ cells, and resulted in the promotion of donor cell engraftment in a minor-mismatched model with $5.5 \mathrm{~Gy}$ preconditioning (50). However, it is known that Tcons and NK cells also express CD25 under inflammatory conditions, for example, following transplantation or in autoimmune diseases. Baeyens et al. observed that low-dose IL-2 combined with rapamycin boosted NK cells and failed to cure type 1 diabetes in a murine model (51). Hirakawa et al. reported that low-dose IL-2 activated STAT5 signaling, not only in CD4 ${ }^{+}$Tregs, but also in a CD56 $6^{\text {bright }} \mathrm{CD} 16^{-} \mathrm{NK}$ cell subset in chronic GvHD patients (18). Mahr et al. demonstrated that IL-2/anti-IL-2 antibody complex injection exacerbated allograft rejection via $\mathrm{NK}$ and $\mathrm{CD}^{+} \mathrm{T}$ cell activation in a mixed chimerism induction model of a fully allo combination with $1 \mathrm{~Gy}$ preconditioning (22). In the current study, WT IL-2 administration increased the proportion of $\mathrm{CD}^{+} \mathrm{T}$ cells, but not that of Tregs. Because the treatment dose of IL-2 in the murine experiments is varied, we also tested a lower dose of WT IL-2 (2500 IU/d) in our model, where we also observed an increase in the percentage of $\mathrm{CD}^{+} \mathrm{T}$ cells (data not shown). In contrast, ortho IL-2-treated mice did not show $\mathrm{CD} 8^{+} \mathrm{T}$ cell increases. WT IL- 2 administration resulted in reduction of BMC signals on BLI on $\mathrm{d} 4$. This rapid BM rejection is thought to be driven by activated NK cells that are resistant in the short term to irradiation (52). In contrast, ortho IL-2 did not interfere with early marrow engraftment, consistent with the absence of NK cell activation after ortho IL-2 stimulation.

The principal mechanism of tolerance induction is thought to be the deletion of alloreactive T cells by donor cells that migrate to the host thymus and perhaps other lymphoid tissues (53). However, in several murine mixed hematopoietic chimerism models with reduced intensity preconditioning, treatment with anti-CD25 
A

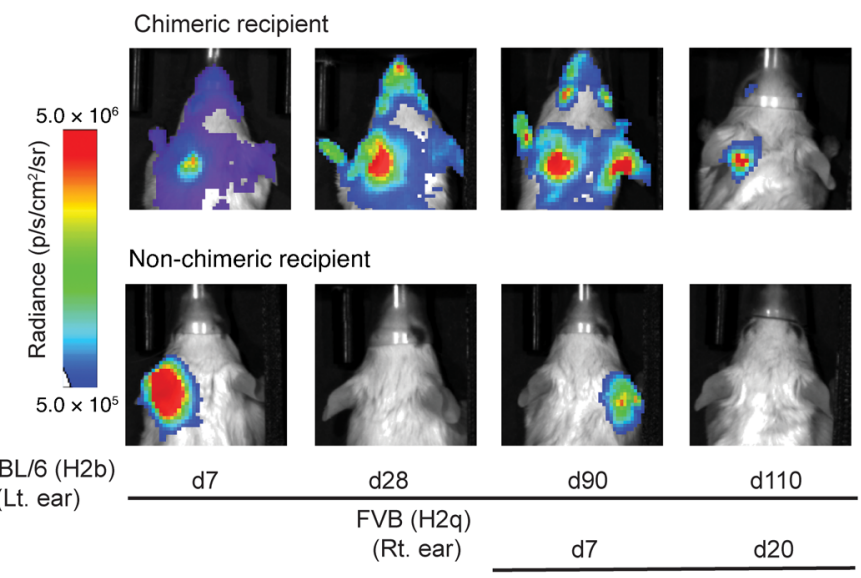

D

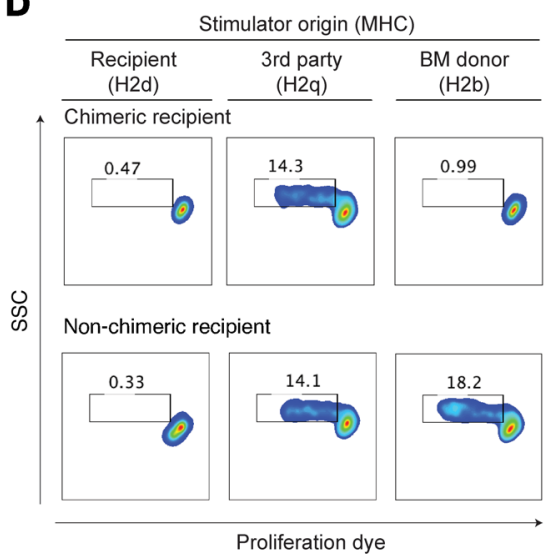

B

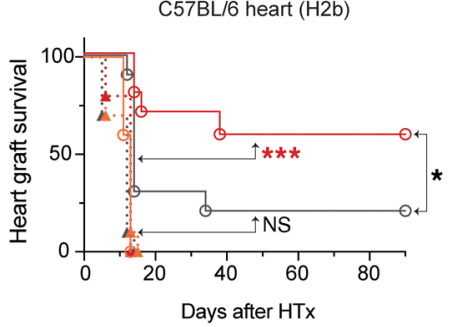

C

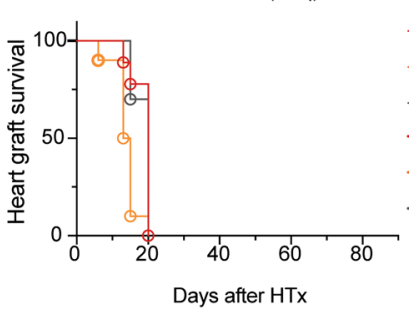

E

O Chimeric $\times$ Non-chimeric PBS A WT IL-2 ๑-Ortho IL-2 + oTreg -WT IL-2 + oTreg - PBS + oTreg A. Ortho IL-2 w/o Treg A. WT IL-2 w/o Treg A. PBS w/o Treg

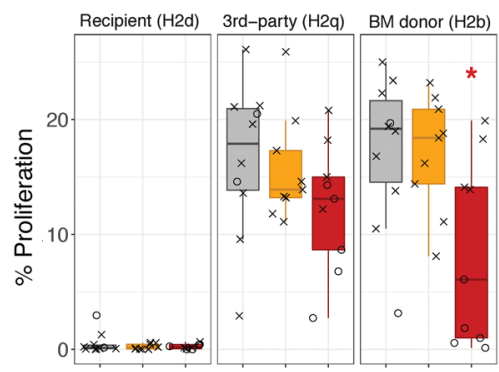

Figure 6. Ortho IL-2 treatment facilitates donor-specific heart transplantation tolerance. Recipients of $\mathrm{H} 2^{\mathrm{b}+} \mathrm{BMT}^{\mathrm{beceived}}$ heart allografts from $/ \mathrm{lu} \mathrm{C}^{\mathrm{H}} \mathrm{H} 2^{\mathrm{b}+}$ or l $\mathrm{CC}^{+} \mathrm{H} 2^{\mathrm{q}+}$ donor mice 2 months after BMT. (A) Representative bioluminescent images show acceptance or rejection of heart allografts in the mice that developed mixed chimerism (chimeric recipient) and in the mice that rejected $\mathrm{H} 2^{\mathrm{b}+} \mathrm{BMCs}$ (nonchimeric recipient). Survival curve for $\mathrm{H} 2^{\mathrm{b}+}$ heart allograft (B) and $\mathrm{H}_{2}{ }^{++}$heart allografts (C) are shown. $P$ values calculated by log-rank test between indicated 2 groups. (D and E) Recipient mice that received oTregs were sacrificed 30 days after $\mathrm{H}^{\mathrm{q}^{+}}$heart transplantation. Negatively isolated T cells were cocultured with host type $\left(\mathrm{H} 2^{\mathrm{d}+}\right)$, third-party type $\left(\mathrm{H} 2^{\mathrm{q}+}\right)$, and $\mathrm{BM}$ donor type $\left(\mathrm{H}^{\mathrm{b}+}\right)$ stimulator cells, and the percentage of proliferating cells was analyzed by flow cytometry on d4. (D) Representative pseudocolor plots showing dilution of proliferation dye. SSC, side scatter. (E) Box plots for percentages of proliferating cells. Dunn's Kruskal-Wallis multiple comparisons calculated among the 3 treatment groups. Pooled data from 2 independent experiments, including 5 mice per group per experiment. ${ }^{*} P<0.05 ;{ }^{* * *} P<0.001$.

$\mathrm{mAb}$, anti-L-2 mAb (54-56), or tacrolimus that abrogates Treg function (57) in the induction phase precluded HSC engraftment, implying that Tregs play a nonredundant role in the induction phase of mixed chimerism when clonal deletion is not completed. This Treg function is not dependent on MHC recognition because adoptively transferred Tregs that were obtained from or primed by a third party retained the ability to facilitate alloengraftment (58). Fujisaki et al. reported that host Tregs colocalized with transplanted syngenic or allogenic HSCs and Treg depletion prevented persistence of donor HSCs in the long term (59). Pierini and Nishikii et al. reported that host Tregs were necessary for B cell lymphopoiesis after HSC transplantation (60). Müller et al. reported that host Tregs facilitate donor HSC engraftment by promoting cell cycling and mobilization of endogenous HSC, thereby making available open niche space for incoming donor HSCs (43). These data collectively suggest that Tregs might have potential to provide a privileged site in the BM niche that protects HSCs from immune attack. In the current study, although the percentages of Foxp $3^{\mathrm{GFP}+}$ Tregs returned to baseline levels 14 days after ortho IL-2 cessation, the majority of chimeric mice maintained donor cell chimerism and established heart allograft tolerance. It has been reported that deletion of Tregs in the maintenance phase does not cause HSC rejection (54-56), implying that the role of Tregs is not essential in the maintenance of hematopoietic chimerism after the establishment of clonal deletion. However, Shinoda et al. reported that transient depletion of both donor- and host-derived Tregs in the maintenance phase of mixed chimerism ( $>100$ days after induction) resulted in skin allograft rejection, whereas hematopoietic chimerism was sustained, suggesting that Tregs are important to maintain tolerance against tissue-specific minor antigens that are expressed on the organ graft but not on HSCs (61). Pilat et al. reported that adoptively transferred Tregs were recruited into the heart allograft and reduced the incidence of chronic allograft rejection $(6,11)$, suggesting the presence of a long-term benefit of adoptive Treg transfer. In the current study, we cannot assess direct impact of ortho IL-2R $\beta^{+}$Tregs on organ graft because heart transplantation was not performed simultaneously with Treg transfer plus ortho IL-2 administration and Foxp $3^{\mathrm{GFP}+}$ Tregs were not observed in the peripheral blood at the time of heart transplantation. Optimal treatment duration in the presence of organ allograft will require further investigation.

We demonstrated here that transducing the ortho IL-2R $\beta$ into host-derived Tregs followed by treatment with the ortho IL-2 cytokine enables the selective stimulation and expansion of Tregs without off-target effects. The selective proliferation of Tregs improved alloengraftment, which was followed by establishment of organ 
transplantation tolerance. Our approach might resolve off-target effects that represent a major limitation for IL-2-based therapies. The limitation of this strategy is that it requires ex vivo manipulation of Tregs, which is not necessary for other IL-2 therapies. However, because the number of Tregs available from patients is limited, ex vivo expansion is helpful for Treg therapy, especially for the patients who are not candidates for toxic cytoreductive preconditioning (e.g., organ-transplant recipients). Furthermore, ortho IL-2R also could be incorporated with other cell-engineering strategies, such as TCR engineering, which endows Tregs' antigen specificity and enhances their immune-suppressive capacity (25). Ortho IL-2 technology might open a new prospect of Treg-based therapy for organ transplantation tolerance.

\section{Methods}

Animals. Eight- to twelve-week-old BALB/cJ mice were purchased from the Jackson Laboratory. FVB/N- and C57BL/6-L2G85 mice that express the luciferase gene $\left(l u c^{+}\right)$were generated as described previously $(62,63)$. We purchased and bred BALB/c mice with a GFP reporter, expressed in conjunction with transcription factor Foxp3 (C.Cg-Foxp $3^{\text {tm2tch }} / \mathrm{J}$; Foxp3 $3^{\text {GFP+ }}$ BALB/c) to facilitate enrichment of highly pure Tregs.

Treg sorting and culture. Single-cell suspensions were obtained from cervical, brachial, axillary, inguinal, and mesenteric lymph nodes and spleens of Foxp $3^{\mathrm{GFP}+}$ BALB/c mice. Following FcR block (Miltenyi Biotec), cells were incubated with Pacific blue-conjugated anti-CD4 (GK1.5), allophycocyanin-conjugated (APC-conjugated) anti-CD8a (53-6.7), and phycoerythrin-conjugated (PE-conjugated) anti-CD25 (PC61.5) (all BioLegend) and using the LIVE/DEAD Fixable Aqua Dead Cell Stain Kit (Thermo Fisher Scientific). After washing, cells were incubated with anti-PE MicroBeads (Miltenyi Biotec), and PE-positive cells were enriched by magnet column isolation. Thereafter, CD $4^{+} \mathrm{CD} 8^{-} \mathrm{CD} 25^{\mathrm{hi}}$ Foxp $3^{\mathrm{GFP}+}$ live cells were purified by FACS using the Aria II (BD). Isolated cells were resuspended into conditioning media (RPMI 1640 supplemented with L-glutamine [2 mM], penicillin $[100 \mathrm{U} / \mathrm{mL}]$, streptomycin $[0.1 \mathrm{mg} / \mathrm{mL}]$, 2-mercaptoethanol $\left[5 \times 10^{-5} \mathrm{M}\right]$, and $10 \% \mathrm{FCS}$ ). Cells were inoculated together with CD3/ CD28 $\mathrm{T}$ cell activation beads (Invitrogen) at a 1:1 ratio in the presence of $1,000 \mathrm{IU} / \mathrm{mL}$ recombinant human IL-2 (hIL-2, teceleukin, Roche) on 96-well flat plates. hIL-2 was replenished on $\mathrm{d} 2$.

Treg transduction. Retroviral supernatant was placed on 24-well plates coated with RetroNectin (Takara). Supernatant was removed after 2 to 3 hours of centrifugation at $2000 \mathrm{~g}$ at $32^{\circ} \mathrm{C}$. Preactivated Tregs were recovered and inoculated into the virus-loaded plates together with refreshed hIL-2 $(1,000 \mathrm{IU} / \mathrm{mL})$ and CD3/CD28 beads ( $1: 3$ cell to beads ratio) and incubated for 72 hours at $37^{\circ} \mathrm{C}$. hIL-2 was replenished on $\mathrm{d} 2$. UT Tregs were cultured on a virus-unloaded plate under the same conditions. Transduction efficiency was determined by tRFP ${ }^{+}$cells by flow cytometry (LSRII; BD).

RNA-Seq. Transduced Foxp3 ${ }^{\mathrm{GFP}+}$ Tregs were inoculated with CD3/ CD28 beads and 1,000 IU/mL hIL-2. Forty-eight hours later, cells were recovered and hEGFR-ortho IL-2R $\beta^{+}$cells were isolated by magnetic isolation. Sorted cells were incubated overnight in the conditioning media without hIL-2. After IL-2 starvation, cells were incubated for 4 hours with $2 \times 10^{4} \mathrm{IU} / \mathrm{mL}$ WT IL-2 or $2 \times 10^{6} \mathrm{IU} / \mathrm{mL}$ ortho IL-2. Control cells were incubated without IL-2 (no IL-2). Total RNA was isolated using the Direct-zol RNA Miniprep Kit (Zymo Research) with oncol- umn DNase digestion. RNA-Seq libraries were prepared using $100 \mathrm{ng}$ of total RNA and the KAPA mRNA HyperPrep Kit (Kapa Biosystems). Each library was indexed using barcoded primers (BIOO Scientific) and was amplified for 10 to 12 cycles. Then, 250 to $350 \mathrm{bp}$ fragments of barcoded PCR products were separated by $2 \%$ E-Gels (Thermo Fisher Scientific), purified by Gel DNA Recovery Kit (Zymo Research), and quantified by Qbit. Equal amounts of each library were pooled and sequenced on an Illumina HiSeq 3000 or NovaSeq platform.

BMT and BLI. WT (CD 45.2 $2^{+}$Thy $\left.1.2^{+}\right)$or Thy $1.1^{+}$congenic marker transgenic (CD45.2 ${ }^{+}$Thy1.1 $1^{+}$BALB/c mice were irradiated at $3.3 \mathrm{~Gy} 4$ hours before $\mathrm{BM}$ cell (BMC) transfer. BM was collected from femurs and vertebraes of $\mathrm{Luc}^{+} \mathrm{C} 57 \mathrm{BL} / 6$ mice. Suspended BMCs were passed through a $70 \mu \mathrm{m}$ mesh, and red blood cells were removed with ACK lysing buffer (Lonza). BM T cells were not removed in this study. BMCs were suspended in PBS and injected at $15 \times 10^{6}$ cells/mouse together with recovered expanded Tregs $\left(1 \times 10^{6}\right)$. Anti-CD4OL (MR1; BioXcell) was diluted with $\mathrm{PBS}$ and injected i.p. at $0.3 \mathrm{mg} / \mathrm{mouse}$ right after BMC transfer. WT or ortho IL-2 MSA was diluted with PBS and injected i.p. at 25,000 IU/dose daily from do to d14. BLI was performed as described previously (43) on d4, d7, d10, d14, d21, and d28 after BMT. Briefly, D-Luciferin Firefly (Biosynth) was injected i.p. 10 minutes prior to acquisition with Ami Imager, and images were analyzed with Aura software (Spectral Instruments Imaging).

Flow cytometry. Six days after BMT, mice were sacrificed and single-cell suspensions were obtained from whole blood, lymph nodes, and spleen. Blood cells were removed with ACK and subsequently incubated with Fc-block reagent, followed by staining with the following antibody cocktails purchased from BioLegend: CD45.1 (clone A20), CD45.2 (clone 104), Thy1.1 (clone OX7), Thy1.2 (clone 53-2.1), TCR- $\beta$ (clone H57-597), CD4 (clone GK1.5), CD8a (clone 53-6.7), CD19 (clone 6D5), CD25 (clone PC61), and ICOS (clone C398.4A). For blood chimerism analysis, blood samples were obtained from the tail tip and suspended into heparin-PBS. After lysins red blood cells, cells were incubated with $\mathrm{H}_{2} \mathrm{~K}^{\mathrm{d}}$ (clone SF1-1.1.1, Thermo Fisher Scientific), H2K b (clone AF6-88.5), CD45.1, CD45.2, TCR- $\beta$, CD4, CD8a, CD19, CD25, Gr1 (clone RB6-8C5), and CD11b (clone M1/70) (all BioLegend). Cells were acquired by LSRII (BD), and data were analyzed with FlowJo software (BD).

All original RNA-Seq data were deposited in the NCBI's Gene Expression Omnibus database (GEO GSE166713) https://www.ncbi. nlm.nih.gov/geo/query/acc.cgi?acc=GSE166713.

Additional methods are provided in Supplemental Methods.

Statistics. Statistical differences were calculated with Welch's 2-sample $t$ test or Mann-Whitney $U$ test for 2-group comparison and Dunn's Kruskal-Wallis test for multiple comparisons by R version 3.6.1. Repeated measures 2-way ANOVA followed by Dunnett's multiple-comparisons test for time-course analysis for BLI and logrank test for heart graft survival were performed using GraphPad Prism, version 8.3.0 (GraphPad Software, LCC).

Study approval. Animal protocols were approved by the Institutional Animal Care and Use Committee of Stanford University.

\section{Author contributions}

$\mathrm{TH}$ conceived and designed experiments. TH conducted the experiments with help from PYL, TLR, FS, MM, and JKL. TLR and PYL performed experiments for the revised manuscript. JB, LLS, and SBW conducted production of retroviral vectors. LKP con- 
ducted production of IL-2 proteins. JXL, PL, and WJL performed and analyzed RNA-Seq data. KS performed and analyzed murine ECG. LLS, BRB, KCG, and RSN helped with critical advice and discussion. TH and RSN wrote the manuscript with contribution from all authors.

\section{Acknowledgments}

This work was supported by grants from the NIH (P01 HL075462 to RSN, UC4DK116264 to KCG, R01 HL11879 and NIAID R37 34495 to BRB, K08 HL151809 to MM, and SIG S10RR027431-01 to Stanford Shared FACS Facility). We also acknowledge support from the Howard Hughes Medical Institute (to KCG, LKP, and LLS) and the Parker Institute for Cancer Immunotherapy (to KCG). TH has received grants from the Japan Society for the Promotion of Science (JSPS) (KAKENHI, Grants-in-Aid for Scientific Research) (15KK0355). Grants were also received from the Geneva University Hospitals Fellowship (to FS), the Swiss Cancer League (BIL KLS 3806-02-2016 to FS), and the Fondation de Bienfaisance Valeria Rossi di Montelera (Eugenio Litta Fellowship to FS). JXL, PL, and WJL were supported by the Division of Intramural Research, National Heart, Lung, and Blood Institute (NHLBI). Next-generation sequencing for the RNA-Seq samples was performed in the NHLBI DNA Sequencing Core. MM received the St. Baldrick's Foundation Scholar Award.
1. Oura T, et al. Chimerism-based tolerance in organ transplantation: preclinical and clinical studies. Clin Exp Immunol. 2017;189(2):190-196.

2. Sprangers B, et al. Origin of enriched regulatory t cells in patients receiving combined kidney-bone marrow transplantation to induce transplantation tolerance. Am J Transplant. 2017;17(8):2020-2032.

3. Kawai T, et al. HLA-mismatched renal transplantation without maintenance immunosuppression. $N$ Engl J Med. 2008;358(4):353-361.

4. Scandling JD, et al. Tolerance and withdrawal of immunosuppressive drugs in patients given kidney and hematopoietic cell transplants. Am J Transplant. 2012;12(5):1133-1145.

5. Taylor PA, et al. L-Selectinhi but not the L-selectinlo $\mathrm{CD}^{4}+\mathrm{CD} 2^{5}+\mathrm{T}$-regulatory cells are potent inhibitors of GVHD and BM graft rejection. Blood. 2004;104(12):3804-3812.

6. Pilat N, et al. T-regulatory cell treatment prevents chronic rejection of heart allografts in a murine mixed chimerism model. J Heart Lung Transplant. 2014;33(4):429-437.

7. Hanash AM, Levy RB. Donor CD4+CD25+ T cells promote engraftment and tolerance following MHC-mismatched hematopoietic cell transplantation. Blood. 2005;105(4):1828-1836.

8. Joffre $\mathrm{O}$, et al. Prevention of acute and chronic allograft rejection with CD4+CD25+Foxp3+ regulatory T lymphocytes. Nat Med. 2008;14(1):88-92.

9. Mahr B, et al. Regulatory T cells promote natural killer cell education in mixed chimeras. Am J Transplant. 2017;17(12):3049-3059.

10. Pilat N, et al. Therapeutic efficacy of polyclonal tregs does not require rapamycin in a low-dose irradiation bone marrow transplantation model. Transplantation. 2011;92(3):280-288.

11. Pilat $\mathrm{N}$, et al. Incomplete clonal deletion as prerequisite for tissue-specific minor antigen tolerization. JCI Insight. 2016;1(7):e85911.

12. Tang Q, Lee K. Regulatory T-cell therapy for transplantation: how many cells do we need? Curr Opin Organ Transplant. 2012;17(4):349-354.

13. Abbas AK, et al. Revisiting IL-2: biology and therapeutic prospects. Sci Immunol. 2018;3(25):eaat1482.

14. Ye C, et al. Targeting IL-2: an unexpected effect in treating immunological diseases. Signal Transduct Target Ther. 2018;3:2.

15. Koreth J, et al. Interleukin-2 and regulatory $\mathrm{T}$ cells in graft-versus-host disease. $N$ Engl J Med. 2011;365(22):2055-2066.
16. Saadoun D, et al. Regulatory T-cell responses to low-dose interleukin-2 in HCV-induced vasculitis. N Engl J Med. 2014;370(8):786.

17. He J, et al. Low-dose interleukin-2 treatment selectively modulates CD4(+) T cell subsets in patients with systemic lupus erythematosus. Nat Med. 2016;22(9):991-993.

18. Hirakawa M, et al. Low-dose IL-2 selectively activates subsets of CD4 ${ }^{+}$Tregs and NK cells. JCI Insight. 2017;1(18):e89278.

19. Spangler JB, et al. Antibodies to interleukin-2 elicit selective $\mathrm{T}$ cell subset potentiation through distinct conformational mechanisms. Immunity. 2015;42(5):815-825.

20. Trotta E, et al. A human anti-IL-2 antibody that potentiates regulatory $\mathrm{T}$ cells by a structure-based mechanism. Nat Med. 2018;24(7):1005-1014.

21. McDonald-Hyman C, et al. Therapeutic regulatory $\mathrm{T}$-cell adoptive transfer ameliorates established murine chronic GVHD in a CXCR5-dependent manner. Blood. 2016;128(7):1013-1017.

22. Mahr B, et al. IL-2/ $\alpha$-IL-2 complex treatment cannot be substituted for the adoptive transfer of regulatory $\mathrm{T}$ cells to promote bone marrow engraftment. PLoS One. 2016;11(1):e0146245.

23. Sockolosky JT, et al. Selective targeting of engineered T cells using orthogonal IL-2 cytokinereceptor complexes. Science. 2018;359(6379):1037-1042.

24. Mackall CL. Engineering a designer immunotherapy. Science. 2018;359(6379):990-991.

25. Raffin C, et al. T reg cell-based therapies: challenges and perspectives. Nat Rev Immunol. 2020;20(3):158-172.

26. Sarkar D, et al. Ex vivo expanded autologous polyclonal regulatory $\mathrm{T}$ cells suppress inhibitor formation in hemophilia. Mol Ther Methods Clin Dev. 2014;1:14030.

27. Li DY, Xiong XZ. ICOS ${ }^{+}$Tregs: a functional subset of tregs in immune diseases. Front Immunol. 2020;11:2104.

28. Takahashi $\mathrm{T}$, et al. Immunologic self-tolerance maintained by CD25+CD4+ naturally anergic and suppressive T cells: induction of autoimmune disease by breaking their anergic/suppressive state. Int Immunol. 1998;10(12):1969-1980.

29. Pandiyan P, et al. CD4+CD25+Foxp3+ regulatory $T$ cells induce cytokine deprivation-mediated apoptosis of effector CD4+ T cells. Nat Immunol. 2007;8(12):1353-1362.

30. Thornton AM, et al. Activation requirements for the induction of CD4+CD25+ T cell suppressor function. Eur J Immunol. 2004;34(2):366-376.

31. Li P, et al. STAT5-mediated chromatin interactions in superenhancers activate IL-2 highly inducible genes: functional dissection of the Il2ra gene locus. Proc Natl Acad Sci U S A. 2017;114(46):12111-12119.

32. Ballesteros-Tato A, et al. Interleukin-2 inhibits germinal center formation by limiting $\mathrm{T}$ follicular helper cell differentiation. Immunity. 2012;36(5):847-856.

33. Zamorano J, et al. Regulation of cell growth by IL-2: role of STAT5 in protection from apoptosis but not in cell cycle progression. J Immunol. 1998;160(7):3502-3512.

34. Yates K, et al. Comparative transcriptome analysis reveals distinct genetic modules associated with Helios expression in intratumoral regulatory T cells. Proc Natl Acad Sci U S A. 2018;115(9):2162-2167.

35. Chen EY, et al. Enrichr: interactive and collaborative HTML5 gene list enrichment analysis tool. BMC Bioinformatics. 2013;14(1):128.

36. Kuleshov MV, et al. Enrichr: a comprehensive gene set enrichment analysis web server 2016 update. Nucleic Acids Res. 2016;44(W1):W90-W97.

37. Rochman Y, et al. New insights into the regulation of T cells by $\gamma$ c family cytokines. Nat Rev Immunol. 2009;9(7):480-490.

38. Wekerle T, et al. Extrathymic T cell deletion and allogeneic stem cell engraftment induced with costimulatory blockade is followed by central T cell tolerance. JExp Med.1998;187(12):2037-2044.

39. Tomita Y, et al. Role of intrathymic clonal deletion and peripheral anergy in transplantation tolerance induced by bone marrow transplantation in mice conditioned with a nonmyeloablative regimen. JImmunol. 1994;153(3):1087-1098.

40. Taylor PA, et al. CD4(+)CD25(+) immune regulatory cells are required for induction of tolerance to alloantigen via costimulatory blockade. J Exp Med. 2001;193(11):1311-1318.

41. Taylor PA, et al. Requirements for the promotion of allogeneic engraftment by anti-CD154 (antiCD4OL) monoclonal antibody under nonmyeloablative conditions. Blood. 2001;98(2):467-474.

42. Sun JC, et al. NK cells and immune "memory". JImmunol. 2011;186(4):1891-1897.

43. Müller AMS, et al. Donor hematopoiesis in mice following total lymphoid irradiation requires host T-regulatory cells for durable engraftment. Blood. 2014;123(18):2882-2892. 
44. Gondek DC, et al. Cutting edge: contactmediated suppression by CD4+CD25+regulatory cells involves a granzyme B-dependent, perforin-independent mechanism. JImmunol. 2005;174(4):1783-1786.

45. Gondek DC, et al. Transplantation survival is maintained by granzyme ${ }^{\mathrm{B}}+$ regulatory cells and adaptive regulatory T cells. J Immunol. 2008;181(7):4752-4760.

46. Barron L, et al. Cutting edge: mechanisms of IL-2-dependent maintenance of functional regulatory T cells. JImmunol. 2010;185(11):6426-6430.

47. Chinen T, et al. An essential role for the IL-2 receptor in $\mathrm{T}_{\text {reg }}$ cell function. Nat Immunol. 2016;17(11):1322-1333.

48. Landuyt AE, et al. Cutting edge: ICOS-deficient regulatory $\mathrm{T}$ cells display normal induction of Il1O but readily downregulate expression of Foxp3. JImmunol. 2019;202(4):1039-1044.

49. Kornete M, et al. ICOS-dependent homeostasis and function of Foxp3+ regulatory T cells in islets of nonobese diabetic mice. JImmunol. 2012;188(3):1064-1074.

50. Shatry A, Levy RB. In situ activation and expansion of host Tregs: a new approach to enhance donor chimerism and stable engraftment in
MHC-matched allogeneic HCT. Biol Blood Marrow Transplant. 2009;15(7):785-794.

51. Baeyens A, et al. Limitations of IL-2 and rapamycin in immunotherapy of type 1 diabetes. Diabetes. 2013;62(9):3120-3131.

52. Raulet DH. Bone marrow cell rejection, MHC, NK cells, and missing self recognition: ain't that peculiar (with apologies to Marvin Gaye). J Immunol. 2015;195(7):2923-2925.

53. Pilat $\mathrm{N}$, et al. Combining adoptive Treg transfer with bone marrow transplantation for transplantation tolerance. Curr Transplant Rep. 2017;4(4):253-261.

54. Bigenzahn S, et al. The role of non-deletional tolerance mechanisms in a murine model of mixed chimerism with costimulation blockade. Am J Transplant. 2005;5(6):1237-1247.

55. Yamazaki M, et al. Different mechanisms control peripheral and central tolerance in hematopoietic chimeric mice. Am J Transplant. 2007;7(7):1710-1721.

56. Hirai T, et al. Clonal deletion established via invariant NKT cell activation and costimulatory blockade requires in vivo expansion of regulatory T cells. Am J Transplant. 2016;16(2):426-439.

57. Katsumata $H$, et al. Evaluation of the impact of conventional immunosuppressant on the establishment of murine transplantation tolerance - an experimental study. Transpl Int. 2019;32(4):443-453.

58. Steiner D, et al. Tolerance induction by thirdparty "off-the-shelf" CD4+CD25+ Treg cells. Exp Hematol. 2006;34(1):66-71.

59. Fujisaki J, et al. In vivo imaging of T reg cells providing immune privilege to the haematopoietic stem-cell niche. Nature. 2011;474(7350):216-219.

60. Pierini A, et al. Foxp $3^{+}$regulatory T cells maintain the bone marrow microenvironment for B cell lymphopoiesis. Nat Commun. 2017;8(1):1-13.

61. Shinoda K, et al. Depletion of foxp3(+) T cells abrogates tolerance of skin and heart allografts in murine mixed chimeras without the loss of mixed chimerism. Am J Transplant. 2014;14(10):2263-2274.

62. Zeiser R, et al. Differential impact of mammalian target of rapamycin inhibition on CD4+CD25+Foxp3+ regulatory T cells compared with conventional CD4+ T cells. Blood. 2008;111(1):453-462.

63. Cao YA, et al. Shifting foci of hematopoiesis during reconstitution from single stem cells. Proc Natl Aca Sci. 2004;101(1):221-226. 